\title{
THE INTERNAL ROTATION PROFILE OF THE B-TYPE STAR KIC 10526294 FROM FREQUENCY INVERSION OF ITS DIPOLE GRAVITY MODES
}

\author{
S. A. Triana ${ }^{1}$, E. Moravyeji ${ }^{1,5}$, P. I. Pápics ${ }^{1,6}$, C. Aerts $^{1,2}$, S. D. Kawaler ${ }^{3}$, and J. Christensen-DalsgaArd ${ }^{4}$ \\ ${ }^{1}$ Institute of Astronomy, KU Leuven, Celestijnenlaan 200D, Leuven, Belgium \\ ${ }^{2}$ Department of Astrophysics/IMAPP, Radboud University Nijmegen, 6500 GL Nijmegen, The Netherlands \\ ${ }^{3}$ Department of Physics and Astronomy, Iowa State University, Ames, IA 50011, USA \\ ${ }^{4}$ Stellar Astrophysics Centre, Department of Physics and Astronomy, Aarhus University, DK-8000 Aarhus C, Denmark \\ Received 2015 February 10; accepted 2015 July 15; published 2015 August 25
}

\begin{abstract}
The internal angular momentum distribution of a star is the key to determining its evolution. Fortunately, stellar internal rotation can be probed through studies of rotationally split nonradial oscillation modes. In particular, the detection of nonradial gravity modes (g modes) in massive young stars has recently become feasible thanks to the Kepler space mission. Our goal is to derive the internal rotation profile of the Kepler B8V star KIC 10526294 through asteroseismology. We interpret the observed rotational splittings of its dipole $\mathrm{g}$ modes using four different approaches based on the best seismic models of the star and their rotational kernels. We show that these kernels can resolve differential rotation within the radiative envelope if a smooth rotational profile is assumed and if the observational errors are small. Based on Kepler data, we find that the rotation rate near the core-envelope boundary is well constrained to $163 \pm 89 \mathrm{nHz}$. The seismic data are consistent with rigid rotation but a profile with counterrotation within the envelope has a statistical advantage over constant rotation. Our study should be repeated for other massive stars with a variety of stellar parameters in order to determine the physical conditions that control the internal rotation profile of young massive stars, with the aim of improving the input physics of their models.
\end{abstract}

Key words: asteroseismology - stars: individual (KIC 10526294) - stars: oscillations - stars: rotation

\section{INTRODUCTION}

Rotation is one of the major components in the computation of stellar evolution models, beginning with the star formation process and lasting until the death of the star (e.g., Maeder 2009, for a recent monograph on the subject). Even in the case of slow rotation, the related dynamical and mixing processes are not negligible as they substantially affect the structure of the star. Unfortunately, direct measurements of the internal rotation profile of stars are not possible. Hence, the inclusion of rotational effects in models relies on uncalibrated theoretical prescriptions.

Fortunately, the oscillation frequencies of a star are affected by its rotational properties (Ledoux 1951). The frequency splitting of the oscillation modes is well understood in the case of slowly rotating stars for which a first-order perturbation method is sufficient to model the frequencies (e.g., Aerts et al. 2010, for an extensive description). In this work, we assume that we are dealing with an unevolved star that does not possess a magnetic field and whose central frequencies of rotationally split multiplets are not affected by slow rotation. Moreover, we assume that the deformation from spherical symmetry due to centrifugal forces can be ignored. In this case, the frequency splitting of the oscillation modes, as measured in the observer's frame, is due to a combination of mode advection and the Coriolis force, and can be computed from the so-called rotational kernels (see Equation (3.356) in Aerts et al. 2010).

Helioseismology provided a very detailed view of the internal rotation profile $\Omega(r, \theta)$ of the Sun for the radial range $r \in\left[0.2 R_{\odot}, 1.0 R_{\odot}\right]\left(R_{\odot}\right.$ denotes the solar radius) and for all

\footnotetext{
${ }^{5}$ Postdoctoral Fellow of the Federal Science Policy Office of Belgium (Belspo).

${ }^{6}$ Postdoctoral Fellow of the Fund for Scientific Research of Flanders (FWO), Belgium.
}

co-latitudes $\theta$, through frequency inversion of the rotational splittings of its hundreds of detected acoustic modes (e.g., Christensen-Dalsgaard 2002; Thompson et al. 2003), a technique that has even found its way to laboratory experiments (Triana et al. 2014). Given that acoustic modes do not have sufficient probing power in the innermost regions, and that the Sun does not reveal gravity modes, it is not possible to deduce the rotational profile for $r \lesssim 0.2 R_{\odot}$.

Currently, we are unable to derive the rotation profiles for distant stars with similar precision as for the Sun, but applications of asteroseismology have allowed us to determine averaged rotation rate ratios $\Omega_{\text {core }} / \Omega_{\text {envelope }}$ from forward modeling of the rotational splitting for a few main-sequence B stars from ground-based monitoring campaigns (Aerts et al. 2003; Pamyatnykh et al. 2004; Briquet et al. 2007), as well as two $\delta$ Sct- $\gamma$ Dor-type hybrid main-sequence pulsators from Kepler space-based photometry (Kurtz et al. 2014; Saio et al. 2015). Moreover, $\Omega_{\text {core }}$ was derived from gravitydominated mixed modes in hundreds of red giants observed with Kepler (Beck et al. 2012; Mosser et al. 2012), while the estimate of their $\Omega_{\text {envelope }}$ is uncertain due to the remaining dominant influence of the core regions on the measured splittings of the pressure-dominated mixed modes. Deheuvels et al. $(2012$, 2014) performed frequency inversions for seven selected subgiants in different evolutionary stages relying on the splitting of their dipole mixed modes. These authors selected profiles representing a linear decrease in the rotation frequency in the core regions, followed by a constant rotation profile in the extended convective envelope, with $\Omega_{\text {core }} / \Omega_{\text {envelope }}$ ranging from 2 to about 20 . Beck et al. (2014) obtained a similar result for the red giant KIC 5006817, which is the primary of an eccentric binary. Only in some of those studies, e.g., Deheuvels et al. (2014), was a statistical model comparison used to evaluate the likelihood of the optimal shape 
of the rotational frequency throughout the stars, and few continuous and discontinuous functions for $\Omega(r)$ were considered. Despite their frequent occurrence in, e.g., geophysics, counter-rotating solutions were considered inappropriate for stars. Independent of that restriction, the Kepler results for $\Omega(r)$ have so far delivered an important calibration for the improvement of evolutionary models for single and binary lowmass stars, given that the theoretical predictions of angular momentum transport result in core rotation rates that are higher by at least an order of magnitude than observed (see, e.g., Eggenberger et al. 2012; van Saders \& Pinsonneault 2013; Cantiello et al. 2014, for the input physics in question). The redistribution and loss of angular momentum during evolution is also required based on the internal rotation properties of white dwarfs derived from both forward modeling and the inversion of their rotationally split $\mathrm{g}$ mode oscillation frequencies (Charpinet et al. 2009; Córsico et al. 2012).

In this work, we provide the first frequency inverted rotation profile of an unevolved intermediate-mass B-type mainsequence star from its rotationally split dipole gravity modes detected in four years of Kepler data. The paper is organized as follows. We summarize the observational data and the resuls of forward seismic modeling in Section 2. In Sections 3 and 4, we examine in detail the rotational kernels and splittings associated with linear rotation models. In Section 5, we present piece-wise two- and three-zone rotation models. Section 6 is devoted to inversion methods, including theory and results. In Section 7, we present our results for the rotation profile based on Monte Carlo simulations. We summarize and conclude in Section 8.

\section{OBSERVATIONAL INPUT AND RESULTS OF FORWARD SEISMIC MODELING}

The first detailed asteroseismic analysis of the mainsequence B star KIC 10526294 was presented by Pápics et al. (2014) and included an estimate of the amount of core overshoot following earlier approaches for main-sequence $\mathrm{B}$ stars with a well-developed convective core. They characterized KIC 10526294 as a slowly rotating SPB star (see, e.g., Aerts et al. 2010 for a definition) exhibiting a series of 19 quasi-equally spaced dipole modes. So far, KIC 10526294 is the only multiperiodic SPB star with unambiguous detection of rotationally split triplets from the Kepler light curve. For this reason, we are able to probe its interior structure to a deeper level than for any other SPB thus far.

For the purpose of detailed seismic modeling, Moravveji et al. (2015) elaborated on optimal frequency error estimation, taking into account the signal-to-noise ratio, sampling, and correlated nature of the Kepler data, following the method by Degroote et al. (2009). This resulted in a correction factor of 3.0 being applied to the formal errors obtained from the nonlinear least-squares fit. We estimated the splitting for each dipole mode as the average splitting between the measured $m=+1$ and $m=-1$ peaks with respect to the central $m=0$ peak. This comes down to considering only the symmetric component of the splittings. The total variance was then estimated as the variance of the symmetric component plus the inter-variance:

$$
\sigma_{\text {Total }}^{2}=\frac{1}{2}\left(\sigma_{-1}^{2}+\sigma_{+1}^{2}\right)+\sigma^{2}\left(\delta_{-1}, \delta_{+1}\right),
$$

where $\delta_{ \pm 1}$ denotes the $m= \pm 1$ splittings and $\sigma_{ \pm 1}$ their individual uncertainties. This results in larger errors for those
Table 1

Symmetric Components of the Observed Rotational Splittings of KIC 10526294

\begin{tabular}{lccc}
\hline \hline $\begin{array}{l}\text { Central Fre- } \\
\text { quency }(\mu \mathrm{Hz})\end{array}$ & $\begin{array}{c}\text { Splitting } \delta_{\text {nlm }} \\
(\mathrm{nHz})\end{array}$ & $\begin{array}{c}\text { Error } \\
\text { Set 1 }(\mathrm{nHz})\end{array}$ & $\begin{array}{c}\text { Error } \\
\text { Set 2 }(\mathrm{nHz})\end{array}$ \\
\hline 5.4655 & 45.28 & 14.72 & 16.54 \\
5.6272 & 29.49 & 0.69 & 8.70 \\
5.7978 & 33.91 & 7.37 & 8.81 \\
5.9873 & 32.64 & 7.47 & 10.33 \\
6.1739 & 41.99 & 4.42 & 7.56 \\
6.3959 & 35.74 & 0.36 & 4.91 \\
6.6200 & 29.43 & 4.06 & 7.38 \\
6.8703 & 30.42 & 0.55 & 8.55 \\
7.1235 & 33.07 & 3.46 & 6.09 \\
7.4213 & 29.99 & 1.00 & 9.41 \\
7.7616 & 41.55 & 15.04 & 17.17 \\
8.1163 & 28.73 & 1.03 & 6.42 \\
8.5036 & 29.50 & 2.59 & 7.75 \\
8.9398 & 28.18 & 3.18 & 7.23 \\
9.4090 & 27.53 & 0.85 & 4.63 \\
9.9115 & 26.11 & 1.67 & 5.82 \\
10.4495 & 26.41 & 7.02 & 10.04 \\
11.0429 & 25.74 & 0.49 & 5.21 \\
11.7293 & 23.32 & 4.55 & 9.34 \\
\hline
\end{tabular}

splittings with larger asymmetric components. Furthermore, as explained by the authors, the error estimates for the triplet components as computed from the Rayleigh limit, and taking into account mode crowding effects, are too large (Pápics et al. 2014, their Figure 8). Nevertheless, we also used those overestimated values based on the argument that they deliver the most conservative upper limit to the true frequency errors. We list both error sets in Table 1 and in this work show that our conclusions on $\Omega(r)$ are essentially independent on the choice of error set. The best error estimates (Error Set 1) are used throughout the main text, while all of the results relying on the errors that were too large (Error Set 2) are treated in Appendix A.

Following an essentially identical approach as in Pápics et al. (2014), Moravveji et al. (2015) were able to find seismic models that match more closely the observed frequencies due to the inclusion of extra diffusive mixing in the stellar envelope, in addition to core overshooting. Such additional mixing was already found necessary for the B3V SPB star HD 50230 (Degroote et al. 2010). The parameters of the two models are given in Table 2 . We base the present work on the best matching Model 1 from Moravveji et al. (2015) but we checked that the main qualitative features of the resulting rotation profiles from inversion do not depend on our choice of seismic model, as long as it is able to reproduce reasonably well the main observed characteristics of the star. We return to this in Section 6.3.

In a non-rotating star, oscillation mode families are characterized by their radial order $n$ and degree $l$, with individual family members corresponding to different values of $m$, the azimuthal wave number, sharing the same eigenfrequency. Rotation lifts this degeneracy. The radial order $n$ of the 19 detected modes of KIC 10526294 was identified by comparing the periods of the observed zonal $(m=0)$ dipole $(l=1) \mathrm{g}$ modes with those predicted by equilibrium models computed with the MESA stellar structure and evolution code (Paxton et al. 2011, 2013) and coupled to the GYRE stellar 
Table 2

Fundamental Stellar Parameters of KIC 10526294 from the Best Matching Theoretical Model (Model 1) Found by Moravveji et al. (2015) and by Pápics et al. (2014) (Model 2)

\begin{tabular}{lcccccccc}
\hline \hline Model & $T_{\text {eff }}(\mathrm{K})$ & $M_{*} / M_{\odot}$ & $R_{*} / R_{\odot}$ & $f_{o v}$ & $Z$ & $X_{c}$ & Age (Myr) \\
\hline 1 & 13000 & 3.25 & 2.215 & 0.017 & 0.014 & 0.627 & 63 \\
2 & 12470 & 3.20 & 2.100 & $<0.015$ & 0.020 & 0.693 & 1.42 \\
\hline
\end{tabular}

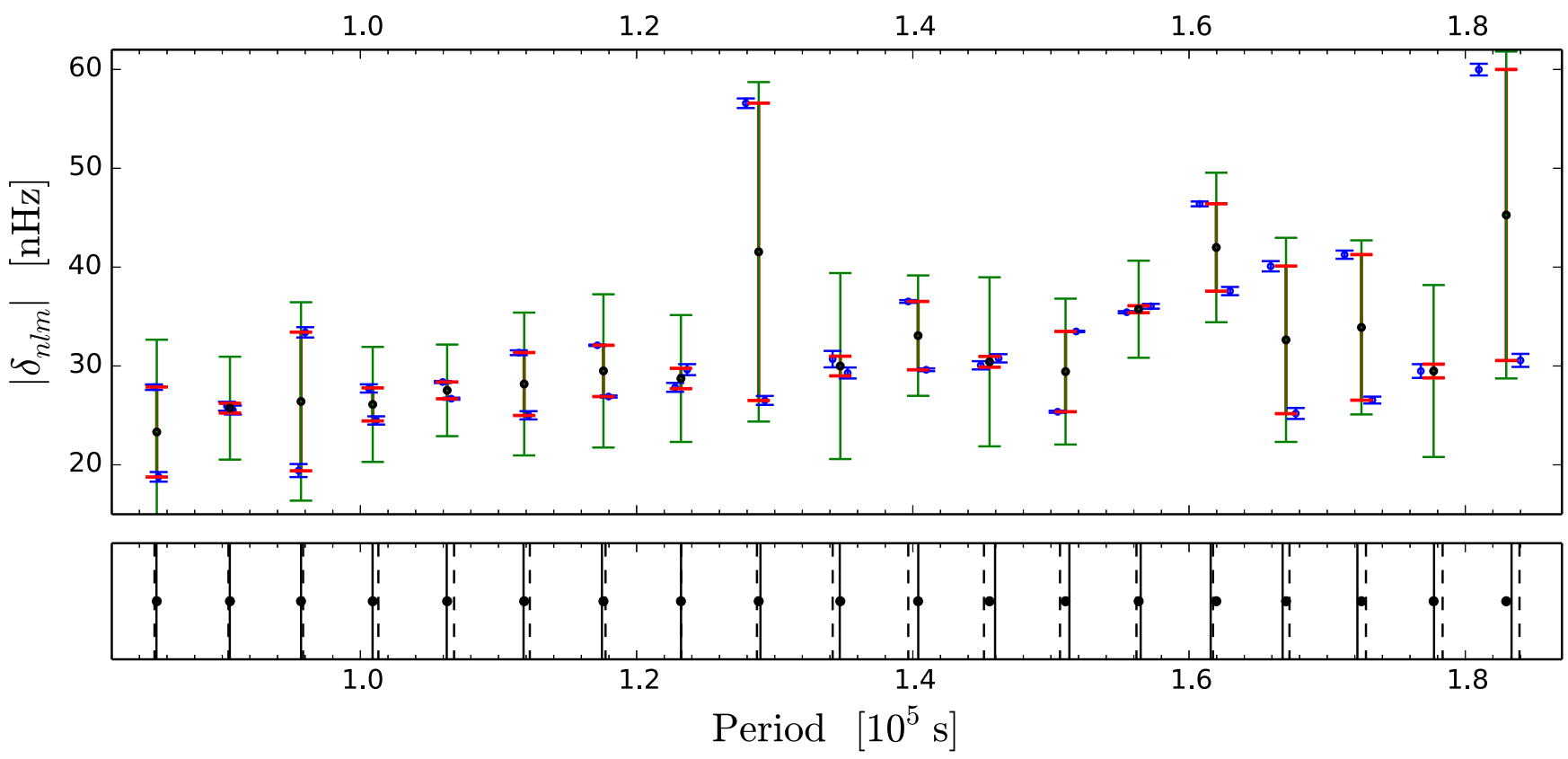

Figure 1. Top: observed rotational splittings (top panel, blue error bars), and the symmetric component used as inversion inputs (top panel, red error bars). The errors on the inversion inputs were taken from Moravveji et al. (2015) and the green error bars are from Pápics et al. (2014); see the text for details. We chose the abscissae of the inversion inputs so as to coincide with the central $m=0$ peak of the observed triplets. Bottom: the location of the central peaks are shown as black dots, and the mode periods from models: vertical solid lines are for the best model in Moravveji et al. (2015; Model 1), the vertical dashed lines are for the best model in Pápics et al. (2014; Model 2).

oscillation code (Townsend \& Teitler 2013). This comparison is possible thanks to the detected triplets on the one hand, and the almost equally spaced sequence of dipole g modes with consecutive order as theoretically expected for such modes on the other hand. The radial orders matching the observations range from $n=32$ to $n=14$.

Most of the 19 dipole modes of KIC 10526294 reveal a very narrow rotationally split triplet structure. If we assume that the cyclic rotation frequency, which we denote as $\Omega$, depends on the radial coordinate $r$ only, then the frequency splitting of a mode with degree $l$ and radial order $n$, denoted as $\delta_{n l m}$, can be written as

$$
\delta_{n l m}=m \beta_{n l} \int_{0}^{R_{*}} K_{n l}(r) \Omega(r) d r,
$$

where the unimodular mode kernel $K_{n l}(r)$ is a function of the mode's displacement amplitudes $\xi_{r}(r)$ (vertical) and $\xi_{h}(r)$ (horizontal), while $\beta_{n l}$ is given by

$$
\beta_{n l}=\frac{\int_{0}^{R_{*}}\left[\xi_{r}^{2}+l(l+1) \xi_{h}^{2}-2 \xi_{r} \xi_{h}-\xi_{h}^{2}\right] r^{2} \rho d r}{\int_{0}^{R_{*}}\left[\xi_{r}^{2}+l(l+1) \xi_{h}^{2}\right] r^{2} \rho d r}
$$

and is connected with Ledoux splitting as $\beta_{n l}=1-C_{n l}$ (e.g., Aerts et al. 2010, Chapter 3). The rotationally induced splittings for the 19 detected modes are shown in Figure 1 (top panel, adapted from Figure 8 in Pápics et al. 2014), together with the mode periods derived from Models 1 and 2 (bottom panel). The mode kernels for both models are shown in Figure 2 and their squared Brunt-Väisälä frequency $N^{2}$ is shown in Figure 3. The latter figure illustrates the slightly more advanced stage of Model 1 compared to Model 2.

Some of the observed splittings are not symmetric with respect to the central $m=0$ peak. These asymmetries are usually related to mechanisms capable of partially lifting the $2 l+1$ degeneracy of a given multiplet, such as deviations from sphericity or large-scale magnetic fields. We assume that we are dealing with a physical phenomenon that causes asymmetries without lifting the degeneracy between the retrograde $(m<0)$ and prograde $(m>0)$ modes. Rotationally induced splittings as expressed by Equation (2) are only capable of lifting the $\pm m$ degeneracy. Hence, forward modeling including only differential rotation up to first order cannot fully capture these observed asymmetries. On the other hand, Equation (2) is still perfectly valid even in the presence of the mechanisms mentioned above, with the caveat that it then accounts for one-half of the splitting between the $+m$ and $-m$ modes and not for the splitting between a mode with a given $m$ and the corresponding central $m=0$ peak. It is known that the presence of a magnetic field near the convective 

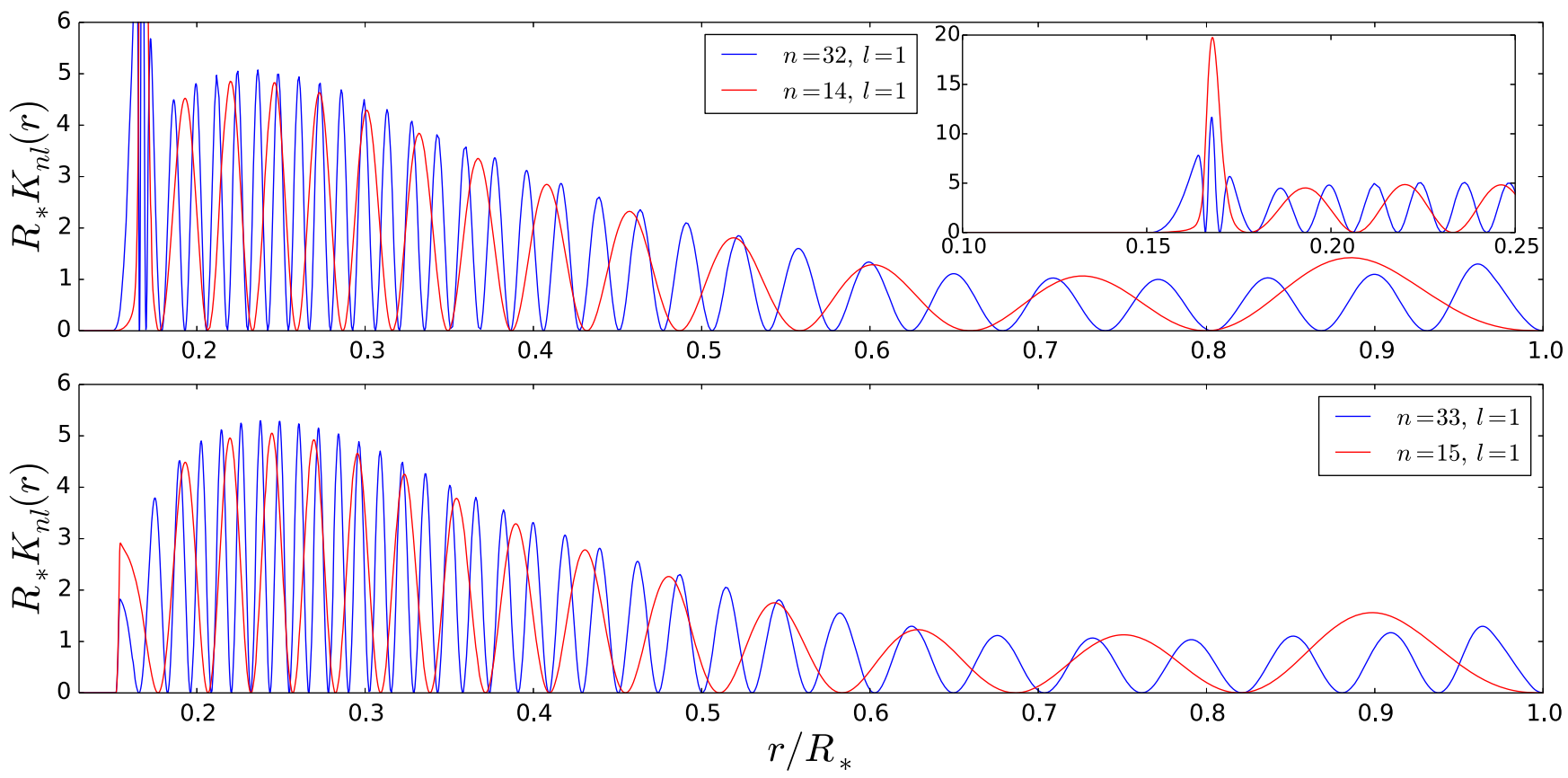

Figure 2. Rotational kernels of dipole zonal modes of highest and lowest radial order, plotted against the stellar fractional radius $r / R_{*}$, for the best-matching models found by (Pápics et al. 2014, lower panel) and (Moravveji et al. 2015, upper panel) - see the lower panel of Figure 1. Some of the modes in the upper panel are trapped near the convective core, as evidenced by the large peak just outside the convective core, as visible in the inset. The kernels from the best seismic model found by Pápics et al. (2014) shown in the bottom panel do not exhibit trapping, but the overall shapes are similar otherwise. Inversion results from both of these models are qualitatively similar.

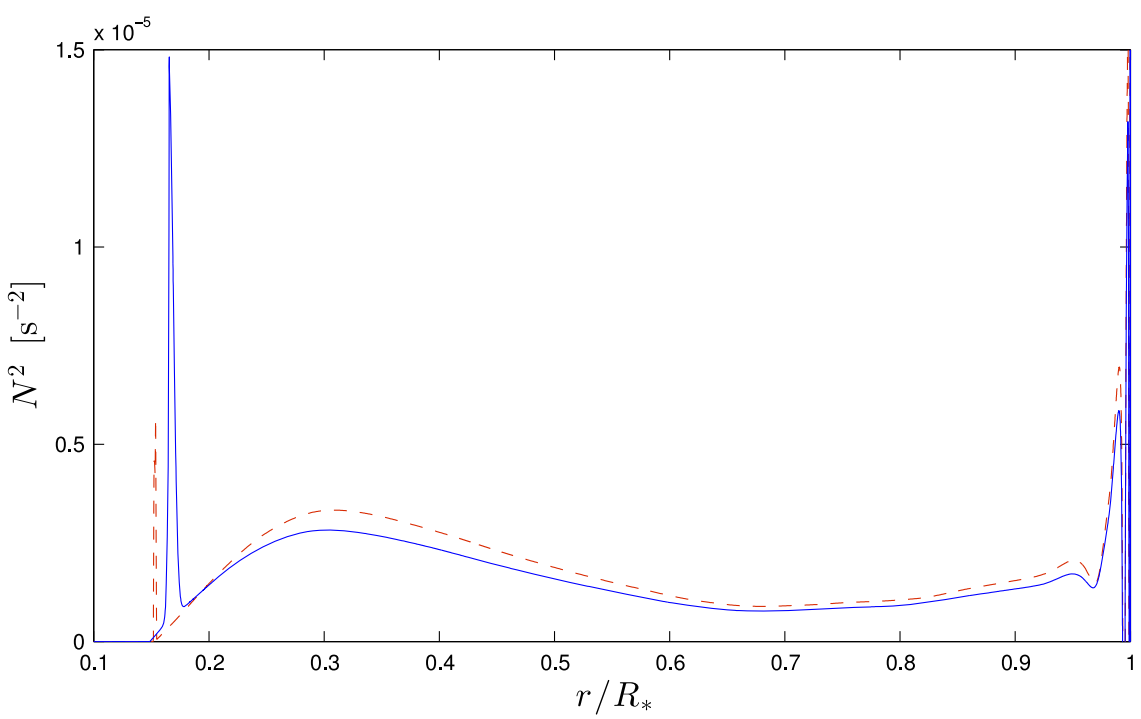

Figure 3. Squared Brunt-Väisälä frequency $N^{2}$ as a function of stellar fractional radius for the best model from Moravveji et al. (2015; continuous blue curve) and for the best model from Pápics et al. (2014; red-dashed curve). The larger peak of the former model near the core boundary (at $r \sim 0.15 R_{*}$ ) is comparatively further away from that boundary than the peak of the latter model, thus allowing some modes to be "trapped" between the core boundary and the peak. See also Figure 2 .

core, as discussed by Hasan et al. (2005), can give rise to such effects. In fact, the mode kernels considered here have substantial amplitudes precisely in that zone, making them particularly susceptible to this effect. Given that we have no information on the presence or absence of a magnetic field in KIC 10526294, we will only address the symmetric components of the splittings in this study, assuming rotation to be the dominant mechanism responsible for the observed splittings and leaving the modeling of the asymmetries for a future, more specialized study. As explained earlier, the presence of the asymmetries leads to increased errors in the splittings.

\section{CUMULATIVE KERNEL INTEGRALS}

Each mode samples differently the internal rotation of the star. This is usually illustrated by plotting the cumulative integral of the kernels $K_{i}$. For increased contrast, in Figure 4, we instead plot the cumulative integral of $k_{i}(r)$, which we define by

$$
k_{i}(r)=K_{i}(r)-\langle K(r)\rangle,
$$

where $\langle K(r)\rangle$ is the average of the kernels across modes at each radius $r$. This $\langle K(r)\rangle$ is the "common" kernel and its integral will only contribute to the average of the splittings. Similarly, 

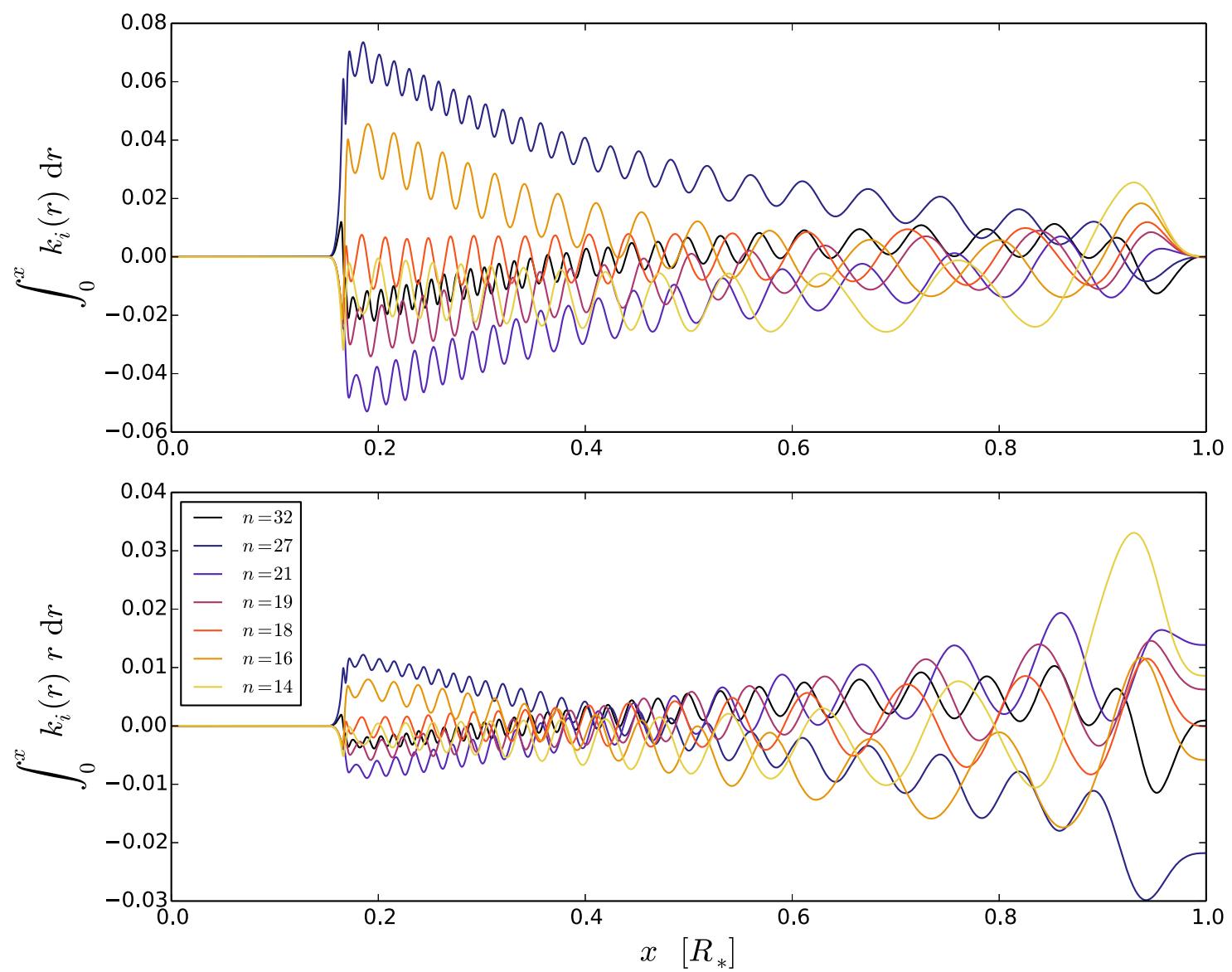

Figure 4. Cumulative integrals of $k_{i}(r)$ (top) and $r k_{i}(r)$ (bottom) for a sample of dipole $(l=1)$ modes from Model 1 with various degrees of trapping. The mode with $n=27$ is the "most" trapped near the core, as compared with the $n=21$ mode, which is the "least" trapped.

we can express a given profile $\Omega(r)$ as the sum of its mean value $\bar{\Omega}$ (across the radial coordinate) plus a fluctuating part $\omega(r)$ (with zero mean):

$$
\Omega(r)=\bar{\Omega}+\omega(r) .
$$

Therefore, we can write the scaled splittings as

$$
\begin{aligned}
\Delta_{i}= & \frac{\delta_{n l m}}{m \beta_{n l}}=\bar{\Omega}+\int_{0}^{R_{*}}\langle K(r)\rangle \omega(r) d r \\
& +\int_{0}^{R_{*}} k_{i}(r) \omega(r) d r .
\end{aligned}
$$

The first two terms on the right-hand side comprise the average splitting, while differences across modes come into play in the last term. Figure 4 shows the cumulative integrals of $k_{i}(r)$ and of $k_{i}(r) r$ for a sample of modes from Model 1 with various degrees of trapping near the core. From the figure, it is evident that the "least" trapped mode (the one with $n=21$ ) would have the largest splitting in response to a rotation profile that increases linearly with radius. Conversely, the "most" trapped mode near the core $(n=27)$ would have the smallest splitting under the same condition.

\section{TRAPPED MODES AND LINEAR ROTATION PROFILES}

Here, we closely follow the analysis by Kawaler et al. (1999) for $\mathrm{g}$ modes in white dwarf pulsators. We take advantage of the fact that some modes are trapped as revealed by the kernel amplitudes. They are trapped very close to the overshooting zone while other modes have comparatively more spread-out amplitudes. This trapping manifests itself as reduced period spacings if we plot them as functions of period (Kawaler \& Bradley 1994). The observed spacings of KIC 10526294 are shown in Figure 5 together with the scaled splittings $\delta_{n l m} / m \beta_{n l}$ (see Equation (2)). A linear fit to the scaled splittings results in a slope of $27.04 \mathrm{nHz} /\left(10^{5} \mathrm{~s}\right)$ and an intercept of $26.20 \mathrm{nHz}$.

We now perform forward modeling to find the predicted splittings using synthetic, linear rotation test profiles. Two test profiles have $\Omega=5 \mathrm{nHz}$ at $r=0$ and have the same slope in absolute value, $1 \mathrm{nHz} / R_{*}$, but are opposite in sign. The results are shown in Figure 6 where the top and bottom plots correspond to increasing and decreasing rotation profiles, respectively. We see the clear signature of mode trapping. Trapped modes are closer to the core, so if the rotation profile increases with radius, then the corresponding splittings are comparatively smaller. Analogously, if the rotation decreases with radius, then the trapped modes will show comparatively larger splittings than the other modes. The latter situation is precisely what we see in Figure 6. A similar situation occurs for the white dwarf PG 1159-035, as reported by Kawaler et al. (1999), where the only difference is that some modes in the white dwarf are trapped close to the surface, such that the results are reversed compared to those for KIC 10526294.

This simplified analysis is helpful because it provides a first idea of the sign of the slope of an unknown rotation profile just 


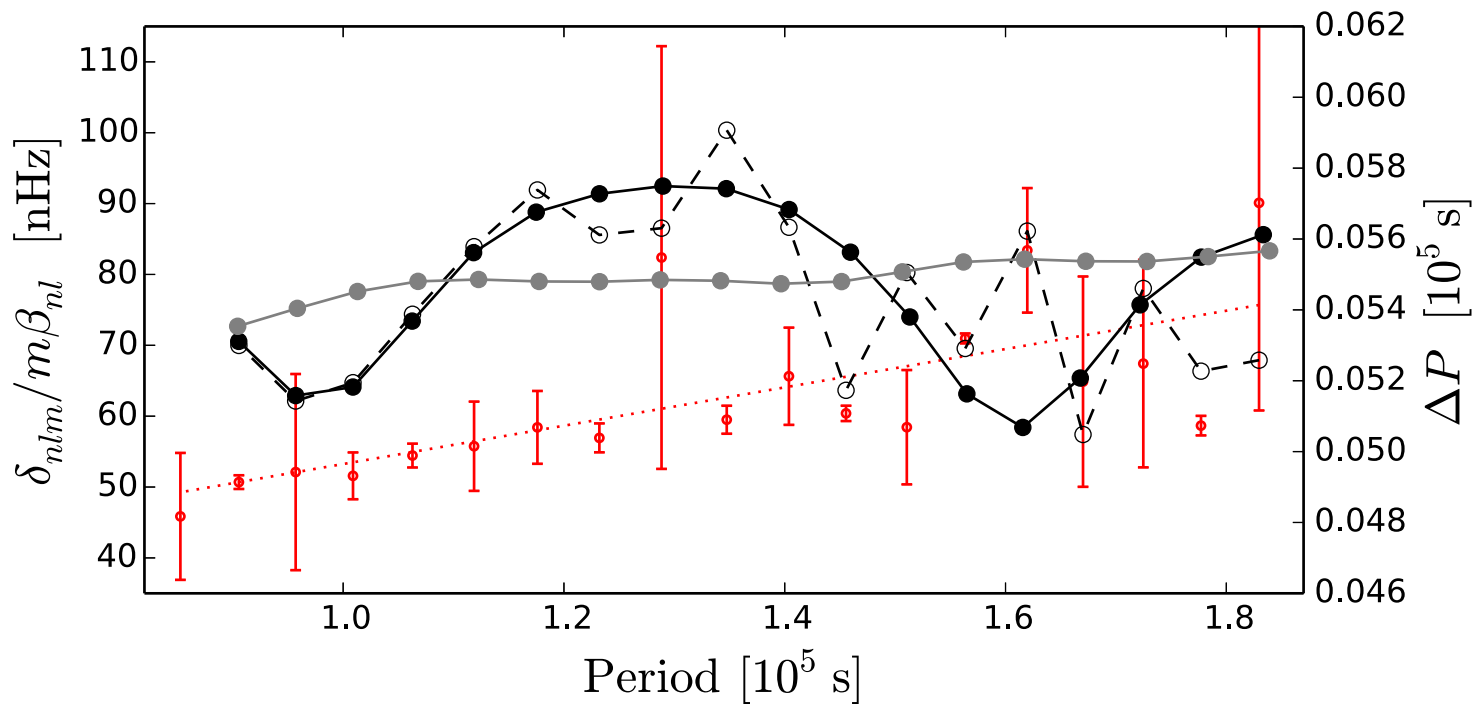

Figure 5. Scaled rotational splittings $\delta_{n l m} / m \beta_{n l}$ deduced from the observations (red error bars) as a function of the mode period together with a linear fit (dotted red line, slope: $27.04 \mathrm{nHz} /\left(10^{5} \mathrm{~s}\right)$, intercept: $26.20 \mathrm{nHz}$, scale is on the left). The dashed line connects the observed period spacings defined as $\Delta P_{n}=\Pi_{n}-\Pi_{n-1}$. The black continuous line connects the spacings derived from the seismic Model 1 in Table 1while the gray continuous line connects the spacings derived from Model 2 (the scale is on the right). The trapped modes of Model 1 are those with periods close to $0.95 \times 10^{5} \mathrm{~s}$ and $1.6 \times 10^{5} \mathrm{~s}$.
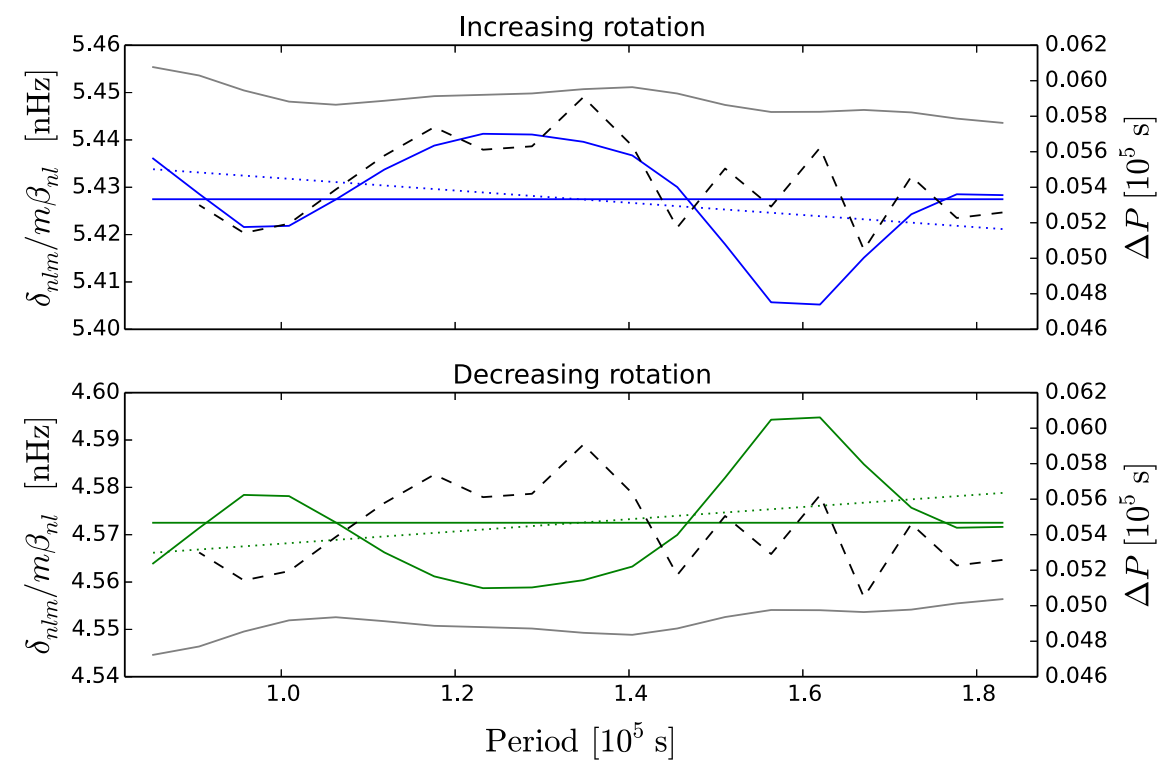

Figure 6. Top: the scaled rotational splittings for a linearly increasing rotation profile derived from Models 1 and 2 (continuous blue and gray curves, respectively), the blue dotted line is a linear fit to the splittings from Model 1; the scale is on the left. Bottom: the splittings for a linearly decreasing rotation profile derived from both Models 1 and 2 (continuous green and gray curves, respectively), the green dotted line is a linear fit to the splittings from Model 1; the scale is on the left. For comparison, the horizontal lines show the splittings from constant rotation profiles that have the same mean splittings as the splittings from the increasing or decreasing profiles (top, blue horizontal line and bottom, green horizontal line, respectively). The black dashed lines on both plots show the period spacings from observation; the scales are on the right.

by plotting the splitings and the period spacings and allows us to see if they vary in phase. In our case, it is not very clear if they vary in phase or not, and so instead we make use of the linear trends of the splittings as indicated with the linear fits (dotted lines) in Figure 6. When period spacings and splittings vary in phase, the linear trend is downward (negative slope). Conversely, when period spacings and splittings are in antiphase, the linear trend is upward (positive slope). The observed splittings of KIC 10526294 have an increasing trend, as shown by the linear fit (dotted red line) in Figure 5. We associate this with a decreasing rotation rate.

We can now perform a simple calculation to estimate the optimal slope $a$ of a linear rotation profile $\Omega(r)=a r+b$ that best matches the observed slope. We assume that the slope of the fit to the measured splittings is linear with respect to the slope of the rotation profile, which seems to be the case. The ratio of the linear slopes associated with the observed splittings and the splittings of the linearly decreasing test profile discussed above is $\sim 2.0918 \times 10^{3}$. Therefore, if we use a linearly decreasing rotation profile with a slope of $2.0918 \times 10^{3}$ times the original slope of our (linearly decreasing) test profile and adjust its mean level to match the mean of the observed splittings, then we might get an idea of the underlying rotation profile. The slope we obtain in this way is $a \sim-2.0918 \mu \mathrm{Hz} / R_{*}$. Adjusting the slope of the test profile to match the trend of the observed splittings also requires 


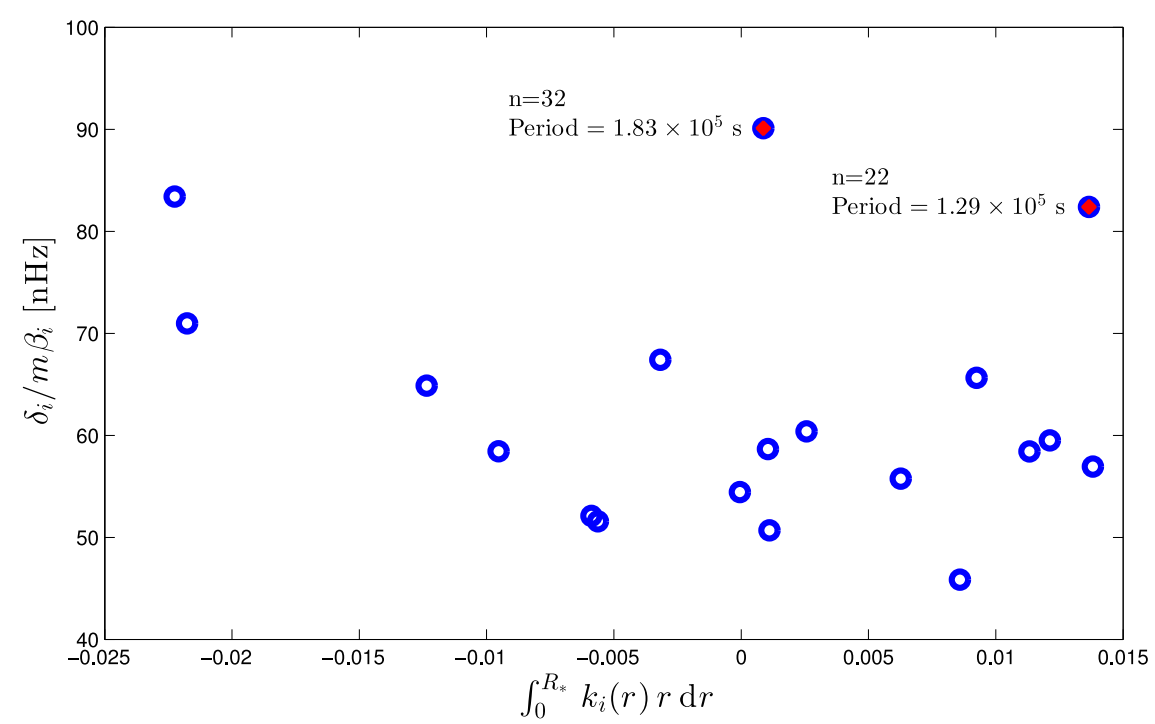

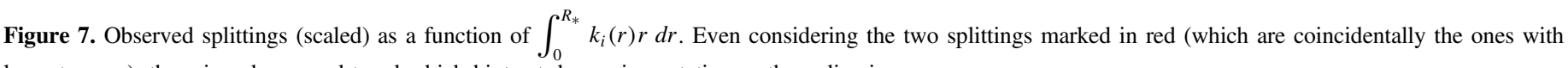
largest errors), there is a downward trend which hints at decreasing rotation as the radius increases.

adjusting $b$ according to Equation (6) if we are to match the average of the observed splittings. We calculate the intercept $b$ as (see also Equation (5))

$$
b=\langle\delta\rangle-a \int_{0}^{R_{*}}\langle K(r)\rangle r d r
$$

where \langle\rangle denotes the average over all of the modes, and we obtain $b \sim 0.957 \mu \mathrm{Hz}$. These values of $a$ and $b$ imply that part of the rotation profile becomes negative. Hence, if the splittings are actually caused by a linear rotation profile or any other profile closely resembling a linear one, then the mean observed splittings should have been considerably higher in magnitude in order to obtain a rotation profile that would not include counter-rotation inside the star. Of course, with this exercise, we were only trying to match the linear trend and the mean of the observed splittings through a linear test profile. In this case, the rms deviation of the predicted splitting from observations is around $22.42 \mathrm{nHz}$, which is relatively large compared to the mean error from the observations, which is $8.41 \mathrm{nHz}$ (scaled, from Error Set 1). We can also use the reduced $\tilde{\chi}^{2}$ values which we compute throughout this work as

$$
\tilde{\chi}^{2}=\frac{\chi^{2}}{\nu}=\frac{1}{\nu} \sum_{i=1}^{M}\left(\frac{\bar{\delta}_{i}-\delta_{i}}{\epsilon_{i}}\right)^{2},
$$

where $\delta_{i}$ and $\bar{\delta}_{i}$ are the measured and predicted splittings, respectively, $\epsilon_{i}$ are the errors, $\nu$ is the effective number of degrees of freedom, and $M$ is the number of observed splittings. In the case just discussed above, we fit two parameters, $a$ and $b$, so that the number of degrees of freedom is $\nu=M-2=17$, which leads to $\tilde{\chi}^{2} \sim 194.8$.

Figure 7 again hints that the rotation is decreasing as the radius increases. We plot the observed splittings as a function of $\int_{0}^{R_{*}} k_{i}(r) r d r$, where $k_{i}(r)$ is defined in Equation (3). This should have been a straight line with a negative slope if the splittings were actually caused by a linear rotation profile. As the figure shows, the splittings have an overall downward trend which is a rough indication that the rotation profile decreases as we move toward the star's surface.

In our next exercise, we search for the linear profile that minimizes $\tilde{\chi}^{2}$. This profile has a slope of $a=-530.7 \mathrm{nHz} / R_{*}$ and an intercept of $b=285.1 \mathrm{nHz}$, leading to $\tilde{\chi}^{2}=6.74$. This profile again leads to negative values for the rotation frequency in the outer envelope. To have an idea of the comparative statistical significance of this result, we computed the optimal constant rotational profile, as well as the optimal linear profile restricted to positive values (using a Lagrange multiplier as an additional fitting parameter). We obtained $\tilde{\chi}_{\text {const }}^{2}=19.86$ and $\tilde{\chi}_{+}^{2}=16.86$, respectively. None of the positive linear rotational profiles have a $\tilde{\chi}^{2}$ similar to the linear profile with counterrotation.

\section{LINEAR, PIECE-WISE ROTATION MODELS}

We now assume a two-zone, piece-wise rotational profile such that its value is $\Omega_{1}$ if $0<r<r_{0}$ and $\Omega_{2}$ if $r_{0}<r<R_{*}$. The parameter $r_{0}$ is variable and we optimize $\tilde{\chi}^{2}\left(r_{0}\right)$ so as to best match the observations, whose errors are derived from Error Set 1 . The resulting $\tilde{\chi}^{2}$ versus $r_{0}$ is shown in the top left panel of Figure 8 . The minimum occurs at $r_{0} \sim 0.166 R_{*}$. The values of $\Omega_{1}, \Omega_{2}$ are shown as functions of $r_{0}$ in the top right panel of Figure 8 . The bottom right panel of Figure 8 shows the observed splittings (scaled) and the splittings for the two-zone model with the minimum at $r_{0}=0.166 R_{*}$, corresponding to $\Omega_{1}=262.71 \mathrm{nHz}$ and $\Omega_{2}=49.33 \mathrm{nHz}$. We note that the boundary of the convective core of Model 1, extended with the core overshoot zone, is situated at $0.1652 R_{*}$ (Moravveji et al. 2015). The second deepest minimum in the top left panel of Figure 8 occurs at $r_{0}=0.741 R_{*}$ and corresponds to $\Omega_{1}=142.0 \mathrm{nHz}$ and $\Omega_{2}=-418.2 \mathrm{nHz}$; this solution leads to an $r_{0}$ that does not play a special role in Model 1 in terms of physical quantities.

The two-zone model thus favors a region rotating with a period of 42 days near the core overshoot zone and a corotating envelope with a period of 254 days $\left(\tilde{\chi}^{2}=1.59\right)$. The second minimum has $\tilde{\chi}^{2}=2.36$ and corresponds to a counterrotating profile. The averaging kernels (for a definition, see 

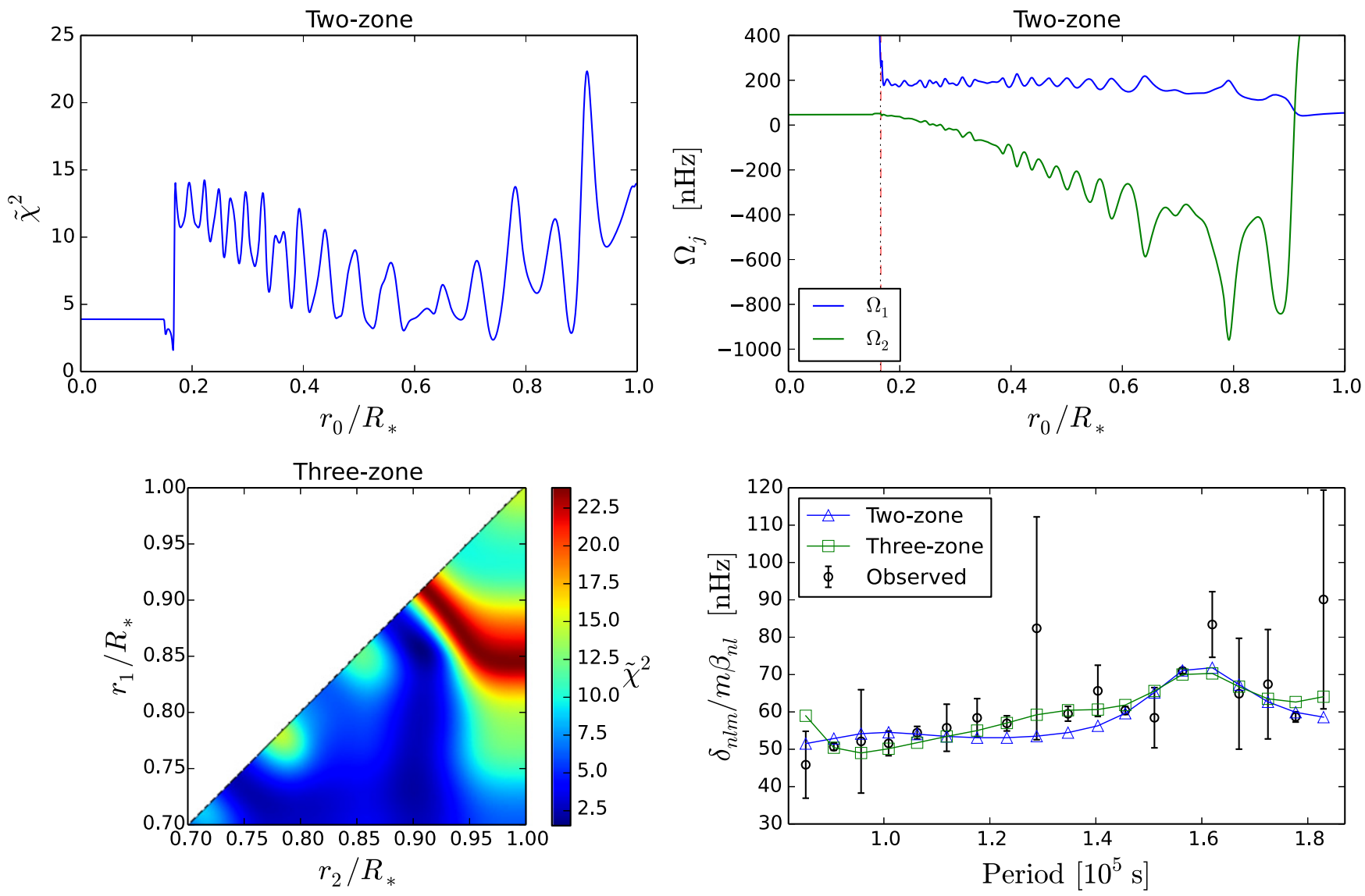

Figure 8. Top left: $\tilde{\chi}^{2}$ from the difference between the predicted and observed splittings as a function of the two-zone parameter $r_{0}$. Top right: the values of $\Omega_{1}$ (blue) corresponding to $r<r_{0}$ and $\Omega_{2}$ (green) for $r>r_{0}$, both as functions of $r_{0}$. The vertical dotted line in black indicates the extent of the core and the overshoot region, it coincides closely with the optimum $r_{0}$. Bottom left: $\tilde{\chi}^{2}$ computed from a three-zone model with $r_{1}$ and $r_{2}$ as parameters. The lowest $\tilde{\chi}^{2}$ is achieved when $r_{1}=0.85 R_{*}$ and $r_{2}=0.91 R_{*}$. Bottom right: observed and predicted splittings from the two-zone model together with the predicted splittings corresponding to the lowest $\tilde{\chi}^{2}$ from the three-zone model (green squares).

Section 6) associated with the best two-zone model are presented in Figure 9 and reveal that the outer zone averaging kernel mostly probes the radiative envelope, while the inner zone averaging kernel exhibits a large maximum just before reaching the core-envelope boundary and rapid oscillations around zero away from it.

A slightly more complex version of this two-zone model is implemented by introducing a third, middle zone where the rotation profile changes linearly from $\Omega_{1}$ at the end of the inner zone to $\Omega_{2}$ at the start of the outer zone. In this case, we have two linear parameters $\Omega_{1}, \Omega_{2}$ and two nonlinear parameters $r_{1}, r_{2}$ defining the zone boundaries. The lowest $\tilde{\chi}^{2}=1.51$ for this three-zone model is achieved when $r_{1}=0.85 R_{*}$ and $r_{2}=0.91 R_{*}$ (see bottom left panel of Figure 8) and indicates counter-rotation $\left(\Omega_{1}=151.8 \mathrm{nHz}, \Omega_{2}=-1069.5 \mathrm{nHz}\right)$. The corresponding splittings are also shown in the bottom right panel of Figure 8.

Very similar results are obtained when the uncertainties are derived from Error Set 2 or when the most asymmetric modes are excluded, as shown by Figures 19 through 21. All of the three-zone $\tilde{\chi}^{2}$ minima in the four cases, i.e., using Error Sets 1 or 2 and with or without the most asymmetric splittings, correspond to cases with counter-rotation. According to their $\tilde{\chi}^{2}$ values, these three-zone models have a statistical significance comparable to the best two-zone model. However, the position of their discontinuities $r_{1}$ and $r_{2}$ have no obvious physical meaning.
To estimate the performance of different models with the goal of choosing the statistically best option, one can only rely on likelihood ratios in the case of nested models, i.e., for models where all of the terms of a simpler model version also occur in a more complex version of the model (e.g., Hastie et al. 2009). We are not in such a situation here because we wish to compare linear, discontinuous linear multi-zone, and continous nonlinear inversion profiles (the latter will be discussed in the next section). In such a case of non-nested models, an adequate statistical measure for model selection is the Akaike Information Criterion (AIC), which assigns a score to a given model rewarding goodness-of-fit (e.g., as measured by $\chi^{2}$ ) but penalizing overfitting, and thus discouraging the use of complex models with too many adjustable parameters (Burnham \& Anderson 2002; Hastie et al. 2009). For our purposes, since we only have a relatively small number of measurements $(M=19)$, it is appropriate to use the corrected AIC (AICc), which we define according to its common use in the literature (e.g., Hurvich \& Tsai 1989):

$$
\mathrm{AICc}=2 k+\chi^{2}+\frac{2 k(k+1)}{M-k-1},
$$

where $k$ is the number of parameters to fit and $M$ is the number of observations. As advocated by Burnham \& Anderson (2002), $k$ should include the variance of the residuals as a parameter to be fitted, e.g., $k=3$ for a linear regression. Based 

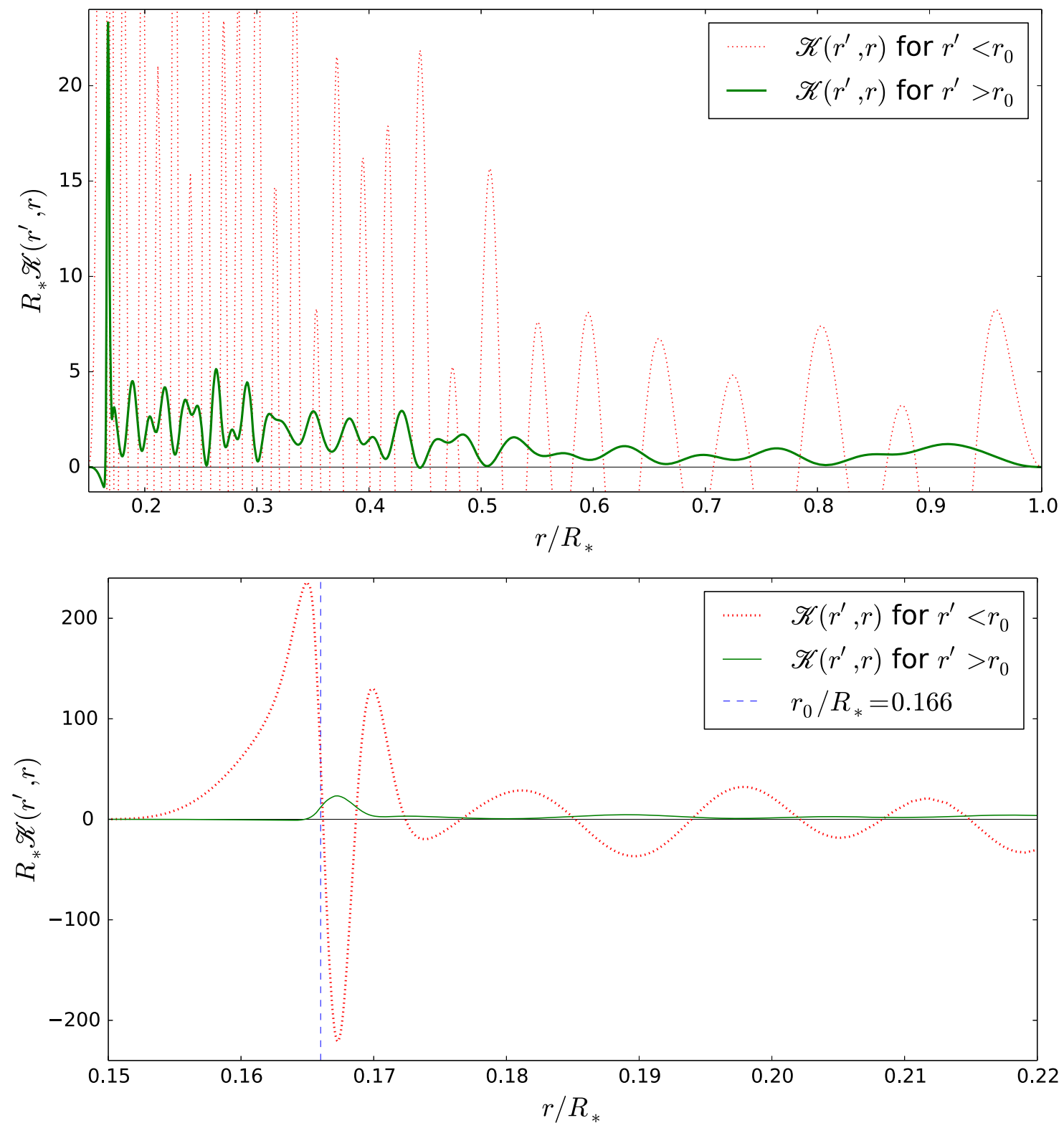

Figure 9. Top: averaging kernels $\mathscr{K}\left(r^{\prime}, r\right)$ of the optimum two-zone model with a discontinuity at $r_{0} / R_{*}=0.166$. Bottom: zoom on the core-envelope boundary region.

on this definition, $k=M-\nu+1$. The preferred model among a set of models is the one with the lowest AICc value, where we limit proper model comparison to the case $M-k>1$. These AICc values are only intended for model intercomparison and have no absolute meaning by themselves. Table 3 lists all the rotation models considered in this work together with some of their associated statistical measures, including their AICc's. Similar tables based on Error Set 2 or on avoiding the most asymmetric splittings are given in Appendix A. We can see from the AICc values in Table 3 that the two-zone piece-wise model outperforms the three-zone model.

We end this section by noting that the overall spectral line broadening of $18 \mathrm{~km} \mathrm{~s}^{-1}$ measured for KIC 10526294, which is the combination of rotational and pulsational broadening (Pápics et al. 2014), is compatible with all of the $\Omega_{2}$-values found from the minima listed in this section and does not allow any discrimination among those solutions, as was the case for the subgiant studied by Deheuvels et al. (2012). In the following, we investigate the rotation profiles obtained from the inversion methods.

\section{INVERSIONS}

First, we introduce the basic concepts and terminology behind the inversion approaches that we applied. We are interested in the approximate determination of $\Omega(r)$ based on a set of observed rotational splittings $\delta_{n l m}$, see, e.g., Gough (1985) for one of the earliest applications of this method in the solar case and Kawaler et al. (1999) and Deheuvels et al. (2012) for applications to white dwarfs and subgiants, respectively. This constitutes a linear problem and the 
Table 3

Comparison of Rotation Profiles for Model 1 (Except for One Entry, where We Used Model 2)

\begin{tabular}{lcrrc}
\hline \hline Rotation Profile & rms Error $(\mathrm{nHz})$ & \multicolumn{1}{c}{$\nu$} & $\tilde{\chi}^{2}$ & AICc \\
\hline Constant & 11.63 & 18.00 & 19.86 & 362.3 \\
Linear & 12.32 & 17.00 & 6.74 & 122.1 \\
Linear+ & 11.46 & 16.00 & 16.86 & 280.7 \\
Two-zone & 10.88 & 16.00 & 1.59 & 36.36 \\
Three-zone & 9.48 & 15.00 & 1.51 & 37.35 \\
RLS,$N=8$ & 9.31 & 14.43 & 0.52 & 24.52 \\
RLS,$N=14$ & 10.46 & 14.32 & 1.44 & 38.18 \\
RLS,$N=8$ (Model 2) $N$ RLS,$N=8$ & 11.35 & 14.74 & 6.42 & 110.3 \\
RLS,$+ N=14$ & 12.05 & 10.43 & 17.16 & 222.2 \\
SOLA, $N=8$ & 11.09 & 5.32 & 3.07 & 184.3 \\
SOLA, $N=14$ & 9.77 & 12.64 & 0.58 & 33.59 \\
\end{tabular}

Note. The Profiles marked with + are enforced to be positive-definite. The effective number of degrees of freedom $\nu$ for the inversions were computed as $M-\kappa$, where $\kappa$ is the trace of the "hat" matrix $\boldsymbol{G} \boldsymbol{C}^{\top}$ (see Section 6), following the same approach as used by Deheuvels et al. (2014) and explained in Hastie et al. (2009). Error set 1 is used here.

approximate solution $\bar{\Omega}(r)$ can be written as

$$
\bar{\Omega}(r)=\sum_{i=1}^{M} c_{i}(r) \Delta_{i},
$$

where $\{i\}$ represents the collective index $\{n l m\}$, $\Delta_{i} \equiv \delta_{n l m} / m \beta_{n l}$ are the scaled splittings, $c_{i}(r)$ are the yetunknown inversion coefficients, and $M$ is the number of observed modes. It is convenient to express the approximate rotational profile $\bar{\Omega}(r)$ in terms of the true profile $\Omega(r)$ by means of the averaging kernels $\mathscr{K}\left(r^{\prime}, r\right)$. They are related to the kernels $K_{i}(r)$ through $\mathscr{K}\left(r^{\prime}, r\right)=\sum_{i=1}^{M} c_{i}\left(r^{\prime}\right) K_{i}(r)$ and fulfill

$$
\bar{\Omega}\left(r^{\prime}\right)=\int_{0}^{R_{*}} \mathscr{K}\left(r^{\prime}, r\right) \Omega(r) d r .
$$

From the preceding relation, it is clear that the averaging kernels $\mathscr{K}\left(r^{\prime}, r\right)$ should be localized around $r^{\prime}$ as much as possible, ideally resembling a delta function $\delta\left(r^{\prime}, r\right)$.

In the following, we consider a radial grid (scaled with the stellar radius $R_{*}$ ) of $N+1$ uniformly spaced points $\left\{r_{0}, r_{1}, \ldots, r_{N}\right\}$ with $r_{0}=0$ and $r_{N}=1$ covering the full range of fractional radius. The goal of the inverse problem is to determine the $N$ unknowns $\bar{\Omega}_{j}$, which represent the predicted angular velocity $\bar{\Omega}$ at radius $r$ such that $r_{j-1} \leqslant r \leqslant r_{j}$, where $j=1, \ldots, N$ is the grid index. This discretization of $\bar{\Omega}$ on a radial grid allows us to write an expression for the corresponding predicted splittings $\bar{\Delta}_{i}$, based on Equation (2), as

$$
\bar{\Delta}_{i}=\sum_{j=1}^{N} G_{i j} \bar{\Omega}_{j}, \text { where } G_{i j}=\int_{r_{j-1}}^{r_{j}} K_{i}(r) d r,
$$

or simply $\bar{\Delta}=\boldsymbol{G} \overline{\boldsymbol{\Omega}}$ in matrix form. Analogously, we express Equation (9) as

$$
\bar{\Omega}_{j}=\sum_{i=1}^{M} c_{i j} \Delta_{i},
$$

with the inversion coefficients $c_{i j}$ constituting the matrix $\boldsymbol{C}$. It is instructive to put the above relation in terms of the discrete version of the true rotation profile $\Omega(r)$. It is straightforward to show that the matrix $\boldsymbol{A}$, defined through $\boldsymbol{A}=\boldsymbol{C}^{\top} \boldsymbol{G}$, accomplishes such a task by fulfilling

$$
\bar{\Omega}_{j}=\sum_{k=1}^{N} A_{j k} \Omega_{k}
$$

Ideally $A_{j k}$ should resemble a Kronecker-delta $\delta_{j k}$ indicating that the recovered profile at a given radius (specified by the grid index $j$ ) does not suffer from "leakage" coming from other radial regions. The matrix $\boldsymbol{A}$ is thus the discrete equivalent of the averaging kernels $\mathscr{K}\left(r^{\prime}, r\right)$.

Note that the observed splittings are linearly related to the predicted splittings through the matrix $\boldsymbol{G} \boldsymbol{C}^{\top}$, which is known as the "hat" matrix. The trace of this matrix is an estimate of the effective number of adjustable parameters (Hastie et al. 2009).

If the observational errors $\epsilon_{i}$ are uncorrelated, as we assume here, then the variance of the recovered profiles can be estimated as

$$
\sigma^{2}\left(\bar{\Omega}_{j}\right)=\sum_{i=1}^{M} c_{i j}^{2} \epsilon_{i}^{2}
$$

The relation above only accounts for the errors on the measurements and its impact on the inversion results; it does not account for the errors inherent to the inversion process itself.

Below, we describe two different inversion techniques that we used to obtain an approximation of the internal rotation of KIC 10526294, as well as quantitative estimates of the uncertainties originating from the measurement errors.

\subsection{Regularized Least-squares Method}

A technique commonly used in helio- and asteroseismology is the regularized least-squares (RLS or Tikhonov) method (e.g., Craig \& Brown 1986), which seeks to minimize the quantity $\mathscr{T}$ defined as

$$
\mathscr{T}=\sum_{i=1}^{M} \frac{\left(\Delta_{i}-\bar{\Delta}_{i}\right)^{2}}{\epsilon_{i}^{2}}+\mu_{\mathrm{RLS}} \int_{0}^{R_{*}}\left(\frac{\partial^{2} \bar{\Omega}}{\partial r^{2}}\right)^{2} d r
$$

where $\Delta_{i}$ are the observed splittings, $\epsilon_{i}$ is the corresponding measurement error, $\bar{\Delta}_{i}$ are the predicted splittings, and $\mu_{\mathrm{RLS}}$ is a free parameter (known as the regularization or smoothing parameter) used to limit the norm of the second derivative on the predicted $\bar{\Omega}(r)$. Using our discrete radial grid described earlier, minimization means $\partial \mathscr{T} / \partial \bar{\Omega}_{j}=0$ for all $j$. This condition can be written more explicitly using Equations (11) and (15) as

$$
\boldsymbol{H}^{\top}(\boldsymbol{G} \overline{\boldsymbol{\Omega}}-\Delta)+\frac{\mu_{\mathrm{RLS}}}{\epsilon_{0}^{2} \delta^{3}} \boldsymbol{L}^{\top} \boldsymbol{L} \overline{\boldsymbol{\Omega}}=0,
$$

where $H_{i j}=G_{i j} / \epsilon_{i}^{2}$, with $\boldsymbol{L}$ being the discrete second derivative operator, $\delta$ the radial grid spacing, and $\epsilon_{0}^{2}$ a scale factor (introduced for convenience) which we set equal to the squared mean of the errors $\epsilon_{i}$. A formal solution is

$$
\overline{\boldsymbol{\Omega}}=\left(\boldsymbol{H}^{\top} \boldsymbol{G}+\frac{\mu_{\mathrm{RLS}}}{\epsilon_{0}^{2} \delta^{3}} \boldsymbol{L}^{\top} \boldsymbol{L}\right)^{-1} \boldsymbol{H}^{\top} \Delta .
$$


Obviously, the inverse matrix on the right-hand side of Equation (17) might not exist and in practice we seek a solution in the least-squares sense instead.

\subsection{The Subtractive Optimally Localized Averaging (SOLA) Method}

The SOLA method (Pijpers \& Thompson 1994) consists of determining a linear combination of the inversion coefficients $c_{i}\left(r^{\prime}\right)$ such that the averaging kernels resemble as much as possible a target function $T\left(r^{\prime}, r\right)$ while keeping the variance of the predicted profiles, $\sigma^{2}(\bar{\Omega})$, low. To implement this method, we minimize

$$
\int_{0}^{R_{*}}\left[\mathscr{K}\left(r^{\prime}, r\right)-T\left(r^{\prime}, r\right)\right]^{2} d r+\frac{\mu_{\mathrm{SOLA}}}{\epsilon_{0}^{2}} \sum_{i=1}^{M} c_{i}^{2}\left(r^{\prime}\right) \epsilon_{i}^{2}
$$

at each $r^{\prime}$ with the constraint $\int_{0}^{R_{*}} \mathscr{K}\left(r^{\prime}, r\right) d r=1$. In addition to the free parameter $\mu_{\text {SOLA }}$, we can also adjust the shape of the target function $T$. The problem reduces to solving the linear set of $M$ equations $(i=1, \ldots, M$ and for each radial location $r^{\prime}$ )

$$
\sum_{k=1}^{M} W_{i k} c_{k}\left(r^{\prime}\right)=\int_{0}^{R_{*}} K_{i}(r) T\left(r^{\prime}, r\right) d r,
$$

where $W_{i k}=\int_{0}^{R_{*}} K_{i}(r) K_{k}(r) d r+\left(\mu_{\mathrm{SOLA}} / \epsilon_{0}^{2}\right) \delta_{i k} \epsilon_{i}^{2}$, together with the constraint $\sum_{k} c_{k}\left(r^{\prime}\right)=1$. Using the discrete radial grid with $N$ segments and choosing the target functions as

$$
T\left(r^{\prime}, r\right)= \begin{cases}N & \text { if } r^{\prime} \in\left(r_{j-1}, r_{j}\right] \\ 0 & \text { otherwise }\end{cases}
$$

the problem to solve becomes

$$
\sum_{k=1}^{M}\left[N\left(\boldsymbol{G} \boldsymbol{G}^{\top}\right)_{i k}+\frac{\mu_{\text {SOLA }}}{\epsilon_{0}^{2}} \delta_{i k} \epsilon_{i}^{2}\right] c_{k j}=N G_{i j},
$$

with the constraint $\sum_{k=1}^{M} c_{k j}=1$. In this case, the target function will approach a Dirac- $\delta$ as $N$ increases. On the other hand, $N$ can be low or moderate as long as $\Omega(r)$ can be assumed not to vary appreciably over a radial segment of the grid.

\subsection{Profiles from Inversion}

We present the internal rotation profiles obtained using the two methods described above. For both the RLS and SOLA methods, we scanned different resolutions ranging from $N=2$ to 14 , each covering a wide range of smoothing parameters $\mu_{\mathrm{RLS}}$ and $\mu_{\mathrm{SOLA}}$, to examine the resulting inversion profiles. To choose the appropriate parameters $N$ and $\mu$ is not an easy task and in practice depends on the a priori information we might have. For example, if we can assume that the rotation profile does not change appreciably over a radial distance $\lambda$, then we can safely use a resolution such that $N=1 / \lambda$. Alternatively, we can interpret the inversion result as providing information only on those components of the rotation profile that do not change appreciably over a distance $1 / N$. Generally, the higher the change of the profile is over a given length scale, the lower the accuracy of the inversion. If we consider that the rotation profile can be represented as a superposition of profiles with increasing detail (e.g., like a Fourier expansion), then the inversion methods can provide useful information at least for those components that change slowly with radius. Below, we discuss the results for the $N$ values with the best statistical score in terms of the AICc.

In Figure 10, we show the inversion profiles with $\mu_{\mathrm{RLS}}=10^{-5}$ and $\mu_{\mathrm{SOLA}}=10^{-2}$, which represent a good balance between the error and resolution for Error Set 1. Similar figures for Error Set 2 and/or ignoring the asymmetric splittings are shown in Figures 22-24 in Appendix A. We see that both methods give qualitatively similar results for $N=8$ and $N=14$. The uncertainty of the SOLA method grows quickly as $N$ increases, as opposed to the RLS method. The averaging kernels, as can be judged from Figures 26 and 27 in Appendix B, are better localized using the SOLA method (as expected, by design), although the uncertainties are somewhat larger compared to the RLS method. The kernels feature larger amplitudes near $r \sim 0.2 R_{*}$, corresponding to small uncertainties at that location. Further out in radius, near $r \sim 0.9 R_{*}$, the uncertainty is larger but the rotation rate is still constrained to be opposite in sign. Note that the kernels provide no information for $r<0.15 R_{*}$ and so the inversion results within this radial range have no meaning (see Appendix B). An appropriate assessment of the inversion's accuracy is provided by the averaging kernels, or their discrete counterpart represented by the matrix A (see Figures 26 and 27). Generally speaking, as $\mu_{\mathrm{RLS}}$ or $\mu_{\mathrm{SOLA}}$ increases, the predicted splittings $\bar{\Delta}_{i}$ will deviate more from the measured ones. The predicted splittings for $N=14$ using both the RLS and SOLA methods and Error Set 1 are displayed in Figure 11. The result in the case of the omission of the asymmetric splittings is provided in Figure 25 of Appendix A.

The best two-zone model from Section 5 might seem to disagree with the profiles obtained from inversion, but upon further inspection they are actually in good agreement, at least for the outer zone. Indeed, if we take the profile from RLS inversion with $N=8, \mu_{\mathrm{RLS}}=10^{-5}$ and interpolate it appropriately, then the resulting average rotation using the outer zone averaging kernel displayed in Figure 9 amounts to $50.34 \mathrm{nHz}$, which is in very good agreement with the $49.33 \mathrm{nHz}$ value obtained in Section 5 for the outer zone. The rotation rate computed from the inversion profile and the inner zone averaging kernel amounts to $206.01 \mathrm{nHz}$, which is to be compared with the $262.71 \mathrm{nHz}$ value for the inner zone in the two-zone model. Although both the best RLS model and the best two-zone are mutually consistent, the RLS model resolves the outer zone better. The AICc values also give preference to the RLS inversions, as we deduce from Table 3.

To add yet another model comparison, we have performed the so-called leave-one-out cross-validation technique (see, e.g., Hastie et al. 2009), which does not rely on $\tilde{\chi}^{2}$ values. This technique consists of omitting one of the measurements when fitting a model and comparing the predicted splitting based on the fitted model $\bar{\Delta}_{i}$ with the measurement that has been omitted $\Delta_{i}: e_{i}=\left(\bar{\Delta}_{i}-\Delta_{i}\right) / \epsilon_{i}$. We performed this procedure omitting one measurement at a time for all measurements and obtained a final score by computing the rms value of all of the $e_{i}$. The preferred model is then the one with the lowest score. In the case of the two-zone model, this score is 1.51 while for the best RLS inversion the score is 0.79 .

Using a synthetic profile without counter-rotation as a test, we found that the RLS inversion method works properly and 

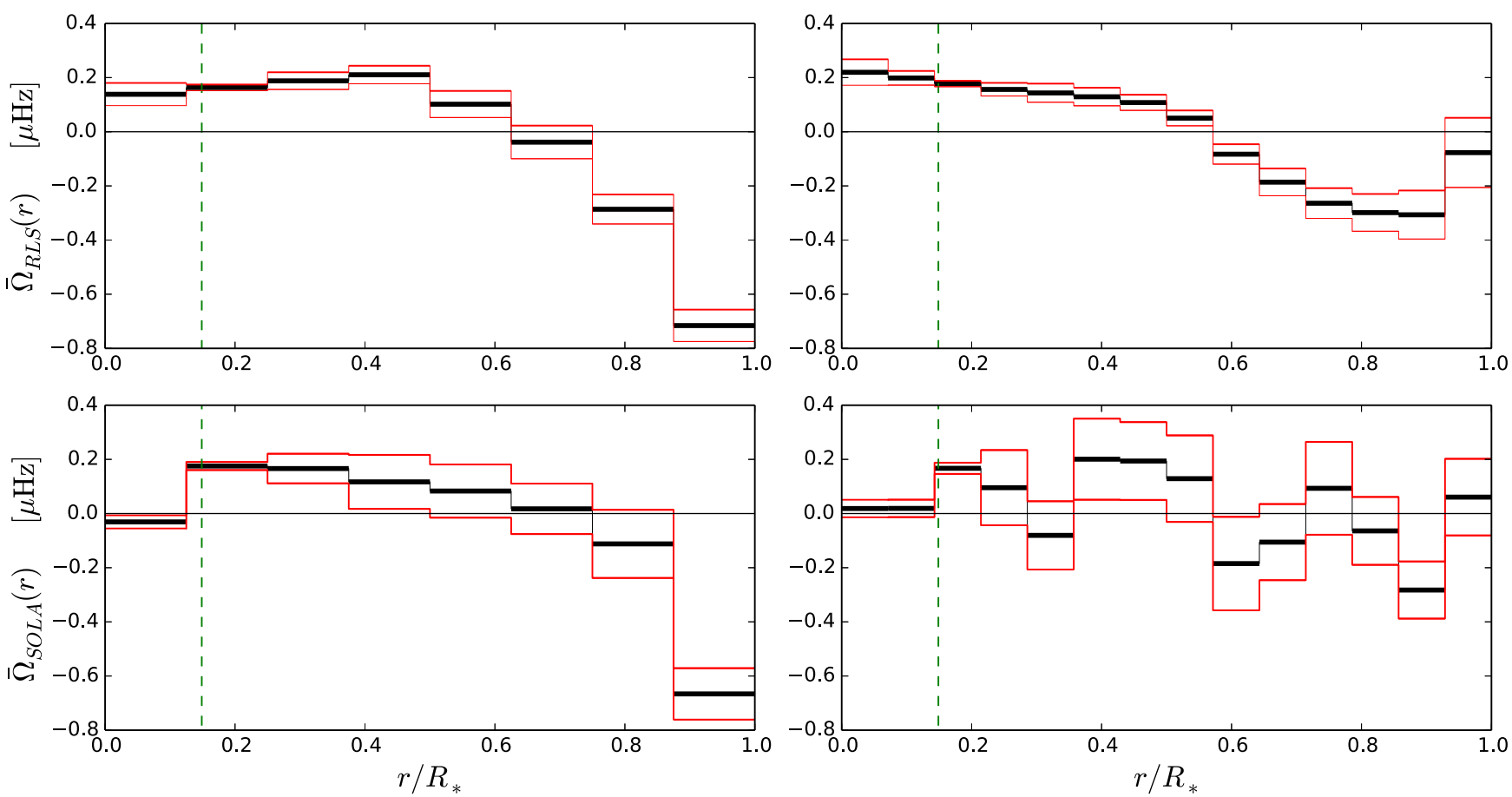

Figure 10. Inversion profiles resulting from the RLS method (top row) with $\mu_{\mathrm{RLS}}=10^{-5}$ and the SOLA method (bottom row) with $\mu_{\mathrm{SOLA}}=10^{-2}$ for two different resolutions $N=8,14$ (left to right). Vertical dashed lines indicate the approximate location of the convective core's radius. Error Set 1 has been used.
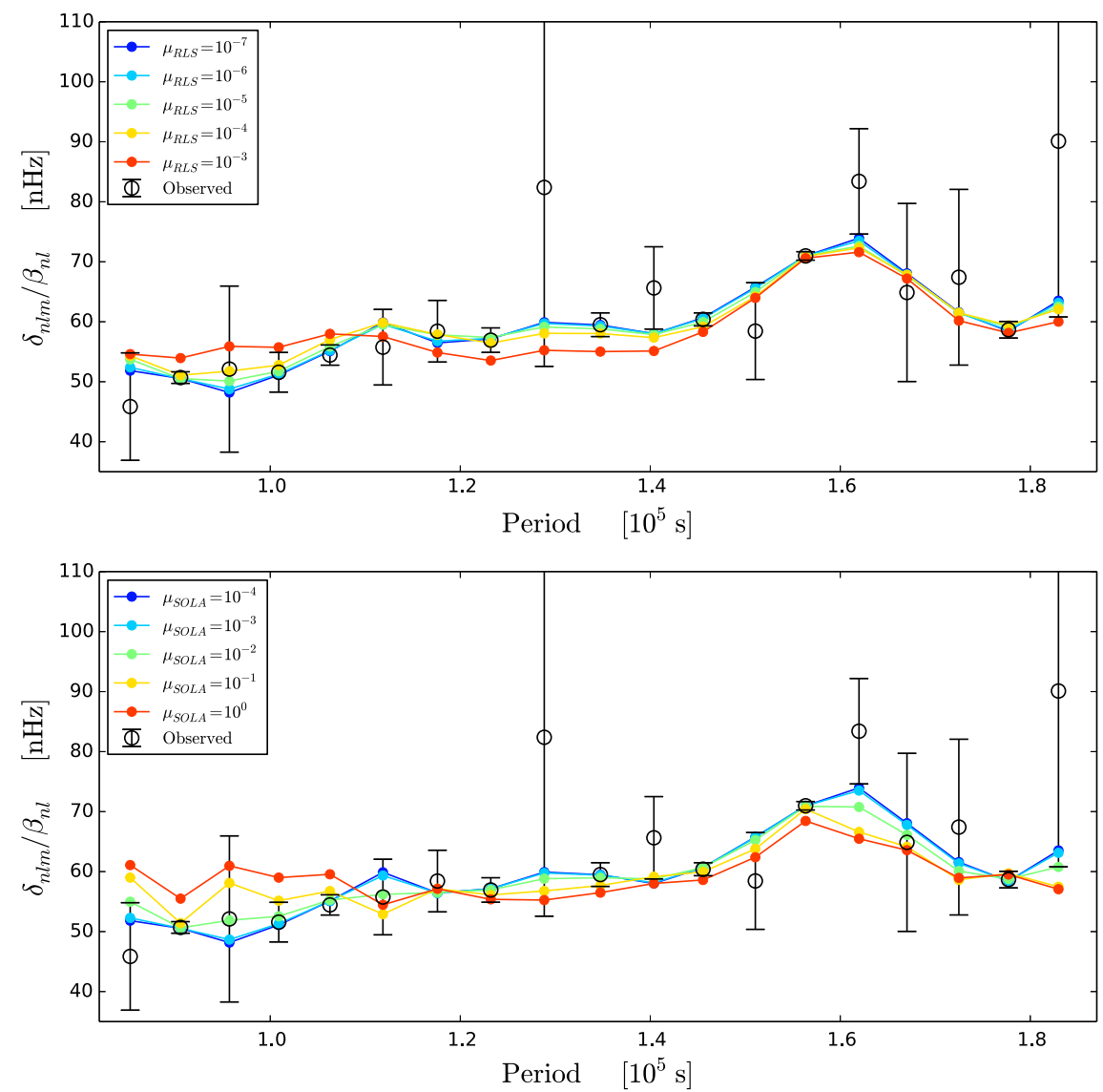

Figure 11. Predicted splittings for both the RLS (top) and SOLA methods (bottom) using a resolution of $N=14$ and a range of $\mu$ parameters for each method. The predicted splittings were computed based on the uncertainties from Error Set 1. 

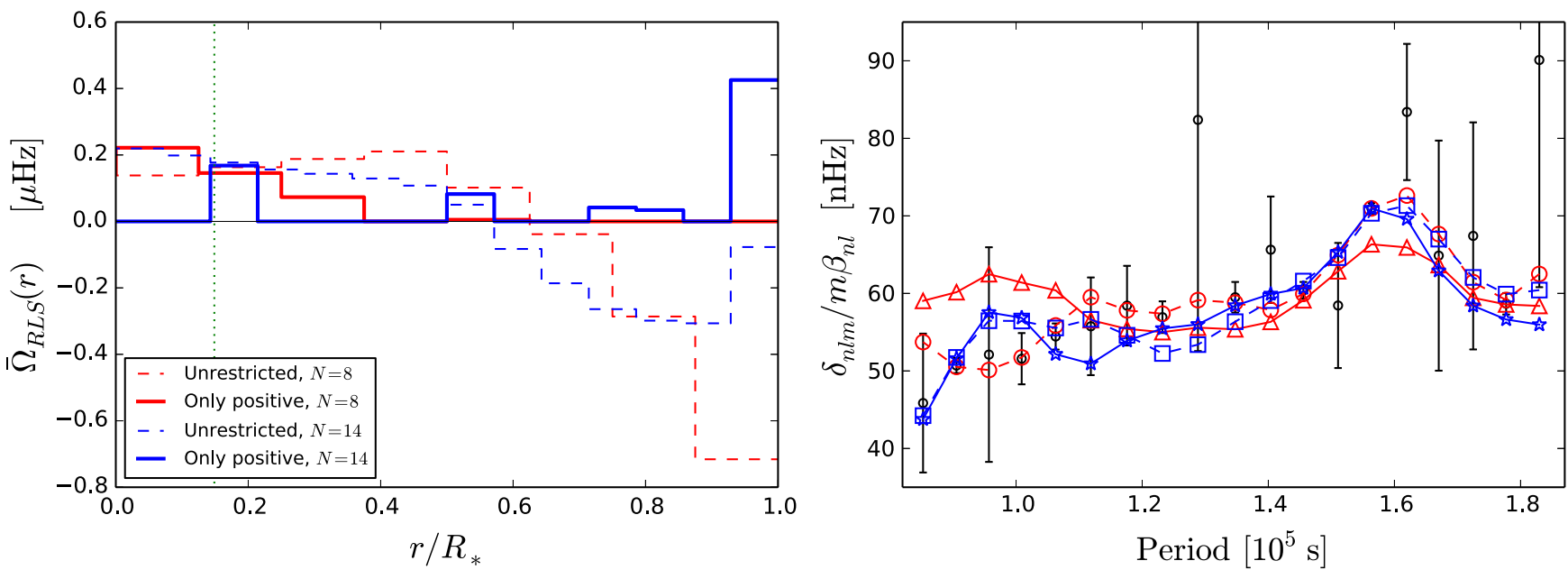

Figure 12. Left: comparison of inversion profiles obtained by restricting the rotation to be positive for all $r$ and those using unrestricted RLS regularization for two different resolutions $N=8,14$ and for $\mu_{\mathrm{RLS}}=10^{-5}$. Right: the corresponding predicted splittings compared to observations. See the main text for details.

that it is not prone to give spurious counter-rotation solutions. See Appendix D for further details.

It is possible to perform RLS regularized inversions enforcing a priori a rotation profile that does not exhibit counter-rotation. We can achieve this by including additional terms to the quantity $\mathscr{T}$ defined in Equation (15) in such a way that they represent our prior knowledge of the rotation profiles being definite positive. Loosely speaking, we can express the probability of obtaining a value $\Omega_{j}$ at a given radial location as being proportional to $e^{\lambda_{j} \Omega_{j}}$, with $\lambda_{j} \geqslant 0$. A concrete technique to solve such a minimization problem is provided by the Karush-Kuhn-Tucker conditions (Kuhn \& Tucker 1951), which consist of minimizing $\mathscr{T}-\sum_{j} \lambda_{j} \Omega_{j}$ together with the constraints $\lambda_{j} \Omega_{j}=0$ for all of the radial locations $j$ (see, e.g., Boyd \& Vandenberghe 2004). The $\lambda_{j}$ are treated as Lagrange multipliers. The result of this exercise is shown in Figure 12.

A qualitative idea of how well these restricted-positive profiles represent the data is provided by the plot on the right of Figure 12. Quantitatively, the $\tilde{\chi}^{2}$ for the restricted-positive profiles are $\tilde{\chi}_{8+}^{2}=112.9$ and $\tilde{\chi}_{14+}^{2}=46.1$, corresponding to resolutions of $N=8$ and $N=14$, respectively. These values are to be compared with $\tilde{\chi}_{8}^{2}=0.52$ and $\tilde{\chi}_{14}^{2}=1.44$ from the unrestricted case. Note here that the effective number of degrees of freedom $\nu$ is reduced considerably when restricting the profiles to be positive-definite given the additional parameters included as Lagrange multipliers. In Table 3, we assembled all of the AICc scores of these and other inversions along with results from previous sections.

Although it is possible to increase the resolution $N$ beyond the number of observations $M$ without overfitting for the inversion result itself, following the principle of regularization (see Appendix C), the value of $\nu$ computed as the trace of the "hat" matrix $\boldsymbol{G} \boldsymbol{C}^{\top}$ might become smaller than unity if the regularization parameters are small, pushing the AICc used for model comparison beyond meaningful values. For this reason, we limit ourselves to moderate resolutions for the sake of meaningful model comparisons. The best models are the RLS or SOLA inversions with $N=8$ with a large statistical margin over all of the other rotational profiles we obtained.
The profiles obtained using the Error Set 2 (Figures 22 and 24) are very similar to those obtained with Error Set 1. Obviously, the inversion uncertainties are larger but still result in very similar counter-rotating profiles. Note that the corresponding $\tilde{\chi}^{2}$ and AICc values (Table 4) give a clear indication of the appreciable error over-estimate in Error Set 2, as already stressed before. Indeed, $\tilde{\chi}^{2}<1$ in virtually all cases. This is already evident from Figure 1 where the point-to-point variation of the rotational splittings is visibly much smaller than the average uncertainty from Error Set 2. The inversion profiles are equally unaffected if the inversion procedure is carried out excluding the most asymmetric splittings as demonstrated by Figures 23 and 24. This is not surprising since the large errors associated with the most asymmetric splittings already give them less weight compared to the others when minimizing $\tilde{\chi}^{2}$.

The RLS inversions can also be carried out by regularizing the norm of the first derivative of the profile or even the norm of the profile itself. It turns out that they are all consistent and have similar properties to the RLS inversions using the norm of the second derivative that we presented earlier. Figure 13 shows the corresponding inversion profiles and their associated uncertainties derived from the measurement errors.

So far, we have based our inversions on Model 1 by Moravveji et al. (2015). However, the main qualitative characteristics of the inversion profiles are robust under different model choices. We have explicitly tested that all the models among the best ones from the forward modeling in Pápics et al. (2014) and Moravveji et al. (2015) produce qualitatively similar results for the inverted rotation profiles, i.e., counter-rotation in the radiative envelope. To provide a specific example, in Figure 14, we show the resulting profile using Model 2 by Pápics et al. (2014). Although the kernels based on this model do not exhibit a large peak right outside the convective core (i.e., no trapped modes), they otherwise have a similar shape (see also Figure 3). Again, the inversion profile hints at counter-rotation within the star's radiative zone. The uncertainty on the recovered profile is somewhat larger overall compared to the uncertainty associated with Model 1 (Figure 10, top left panel). We attribute this to the lack of variation of the kernels in this model, i.e., there are no trapped 


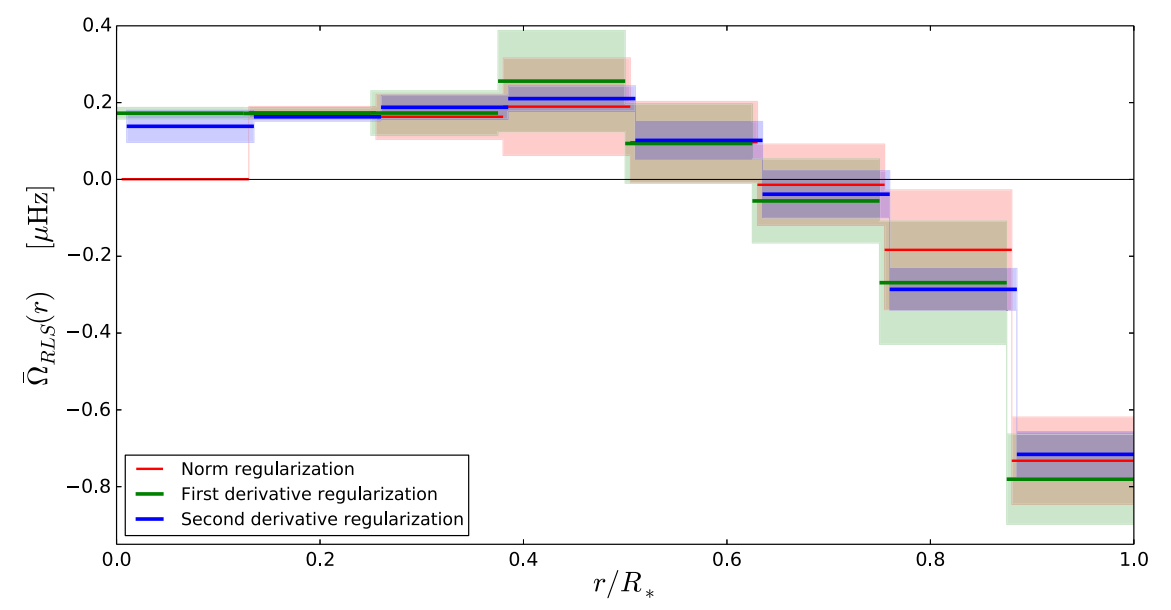

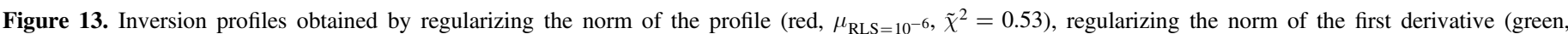
$\mu_{\mathrm{RLS}=10^{-6}}, \tilde{\chi}^{2}=0.51$ ) and regularizing the norm of the second derivative (blue, $\mu_{\mathrm{RLS}=10^{-5}}, \tilde{\chi}^{2}=0.52$ ). For visual aid, the profiles have been slightly shifted horizontally with respect to each other.

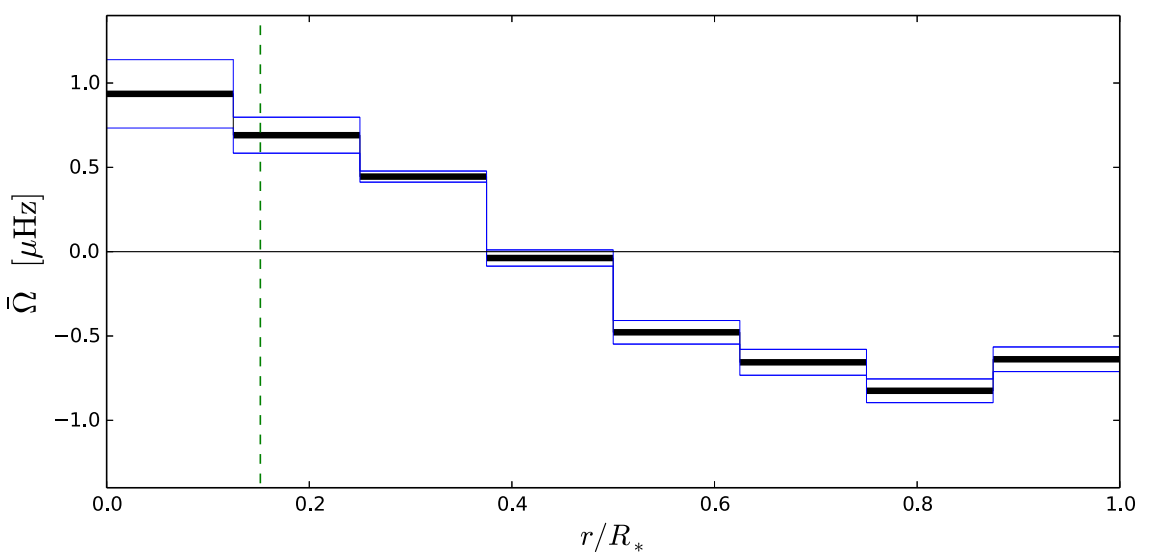

Figure 14. Inversion profile obtained using kernels from Model 2 as found by Pápics et al. (2014). Here, the parameters are identical to those used for the top left panel in Figure $10\left(\mu_{\mathrm{RLS}}=10^{-5}, N=8\right)$. The vertical dashed line marks the convective core radius. The counter-rotation within the radiative zone is a characteristic feature of all the models we examined.

modes to make the kernels more "different" from mode to mode. The more similar kernels for the modes of Model 2 result in higher $\tilde{\chi}^{2}$ and AICc values, which we have added for the case of RLS and $N=8$ in Table 3 .

\section{MONTE CARLO SIMULATIONS}

Yet another method for obtaining an approximation of the real profile $\Omega(r)$ under the assumption of a smooth profile involves generating a large collection of random, synthetic test profiles and assigning a score to each, measuring how close the predicted splittings are to the observed ones. This method is straightforward but very inefficient computationally since the number of synthetic profiles that need to be calculated is necessarily large.

A random, synthetic profile on a radial grid with $Q$ points can be generated by choosing a random rotation value $\Omega_{j}$ at each radial location $r_{j}$. The rotation values are to be picked from a random (uniform) distribution extending from $-h$ to $h$ (in $\mathrm{nHz}$ ), where the range $h$ is to be chosen appropriately as described below. In general, such a profile will exhibit strong fluctuations along the radius, i.e., it will be a "noisy" spiky profile, particularly if $Q$ is large (we used $Q \geqslant 100$ in our simulations). There are a number of ways to smooth out the profile, a simple one being to use a "low pass" filter to remove the "high frequency" components of the profile (if we think of it as a time series). The filter cutoff point defines a characteristic length scale $\lambda$ below which the profile can be considered to have only smooth variations. Some padding at each end of the profile is necessary to avoid end effects when filtering. See Figure 15 for an illustrative example. Once the profile has been smoothed out, it will have spatial fluctuations only on length scales larger than $\lambda$. Since generally $Q>N$, i.e., the random profiles are defined on a finer grid than the one used to define $\boldsymbol{G}$, we should resample the profile to match the radial grid with $N$ points. We do this by calculating the integral average of the random profile along each segment in the radial grid associated with $\boldsymbol{G}$ after an appropriate interpolation. Other methods can be used to achieve the same result. What is essential here is that the smoothing should be chosen in agreement with the final resolution $N$ so that $\lambda N \geqslant 1$.

Once the smoothing is performed, the rotation values are no longer distributed uniformly on the $[-h, h]$ interval, resembling instead a Gaussian distribution. This is simply because we have introduced short-range correlations with our smoothing. Therefore, we should adjust the range $h$ to ensure that the 


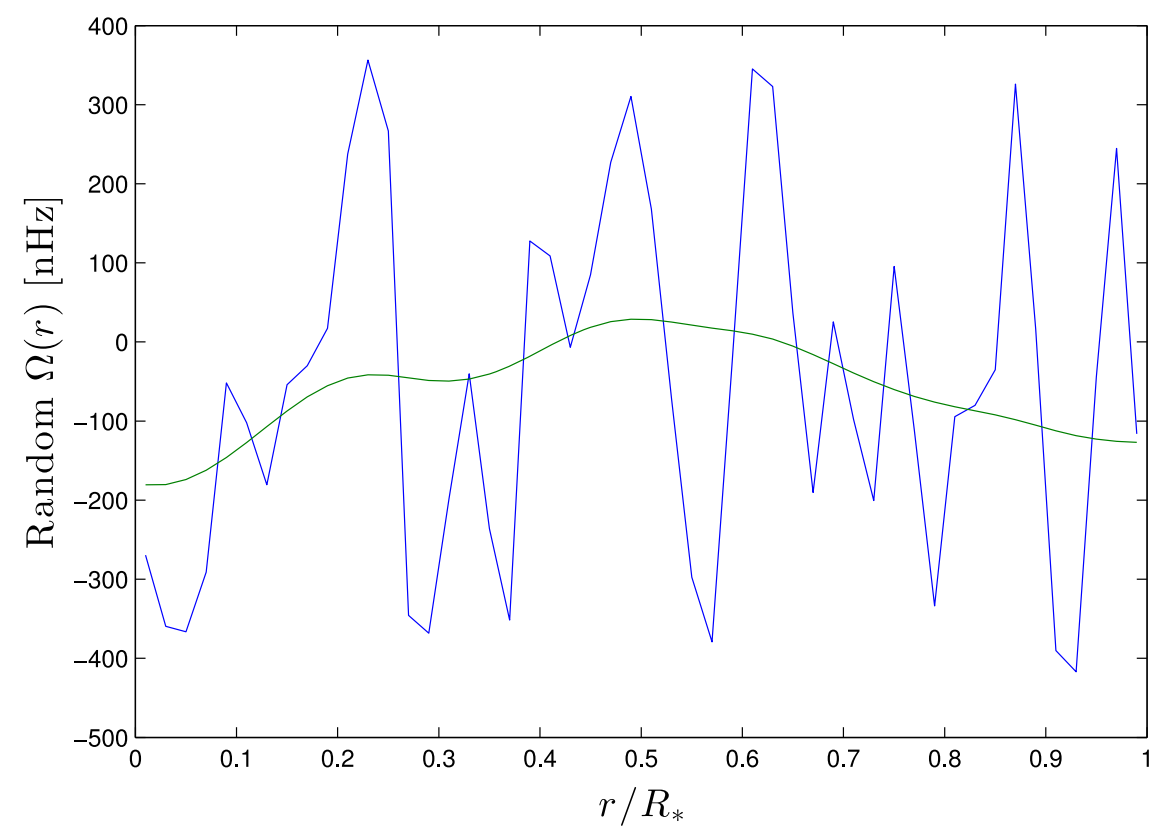

Figure 15. Random profile (blue) before any smoothing with $Q=100$ and $h=500 \mathrm{nHz}$. The "low pass" filtering produces the green curve. The correlation length is $\lambda=0.3$.

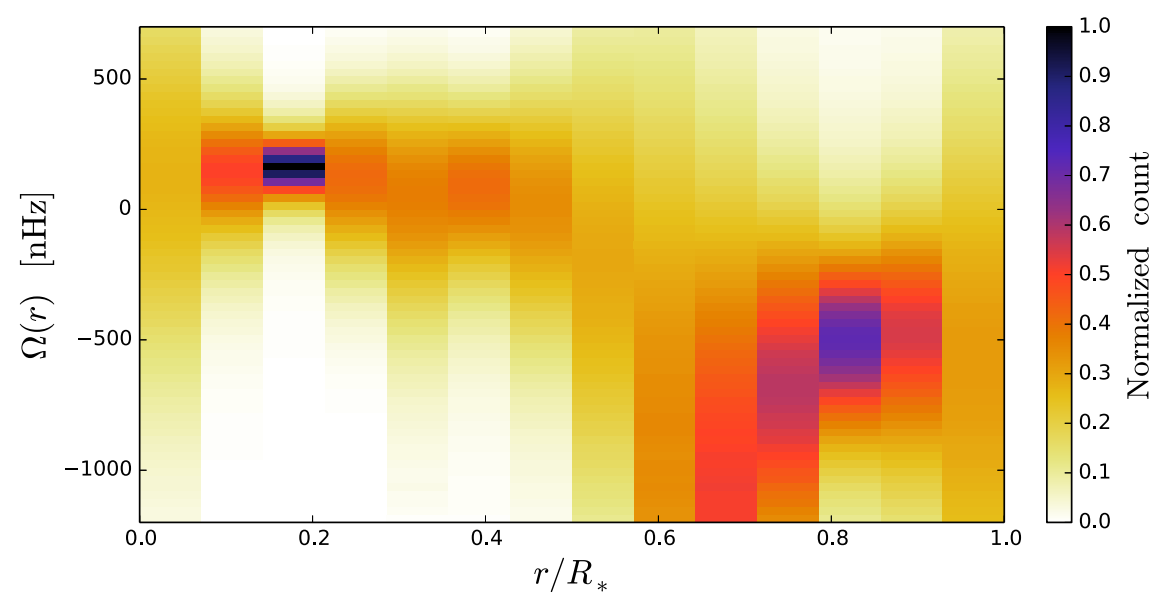

Figure 16. Color coded weighted histogram count for each radial location of a large sample of random rotation profiles using $1 / \chi^{4}$ as weights (see text for details). The original random profiles have been smoothed out so that the typical length scale is $\lambda=0.3$.

rotation values at a given radial location are more or less equally probable, thus uniformly covering the expected range of $\Omega(r)$. This expected range can be roughly estimated from the mean of the observed rotational splittings $\left\langle\delta_{i} / m \beta_{i}\right\rangle$. As a concrete example, we found that for $\lambda=0.3$ and $h=4 \mu \mathrm{Hz}$, the random profiles visited more or less uniformly the range $[-0.2,0.2] \mu \mathrm{Hz}$ within a $14 \%$ margin.

If we interpret the rotation value $\Omega_{j}$ at each radial location as a random variable, and given a large collection of random rotation profiles, then it is possible to calculate the associated covariance matrix. A given row $j$ of this matrix will resemble a Gaussian distribution centered at $r_{j}$. The mean of the FWHM of the Gaussians in all rows is then an (after-the-fact, of course) estimate of $\lambda$. In practice, the random profiles can be considered to remain approximately constant over radial scales not larger than $\sim \lambda / 3$.

Once a random profile has been generated and smoothed out, its associated splittings are calculated via Equation (2). We then compute a score which is proportional to $\chi^{-4}$ (using Error Set 1). The lower $\chi^{2}$ is the higher the score becomes. After scoring a large number of profiles $\left(10^{9}\right)$, we compute the histogram of the rotation rates at each radial location weighted with their corresponding scores and then normalized by an ordinary histogram count. In this way, for each radial location and each rotation rate interval, we obtain a number indicative of its likelihood to explain the observed splittings.

For the results shown in Figure 16, the random profiles have a resolution of $N=14$ and have been smoothed so that $\lambda=0.3$. At each radial segment $r_{j}<r<r_{j}+1$, we computed the weighted histogram of the ocurrences of $\Omega_{j}$ (as explained above) over an interval starting at -1500 and going to $1200 \mathrm{nHz}$, subdivided into 71 bins. By comparing Figures 10 and 16, we see that the Monte Carlo method reproduces very well the rotation rates at $r \sim 0.2 R_{*}$ while giving only a broad distribution of rotation rates centered around negative values at $r \sim 0.8 R_{*}$. We note that virtually none of the high scoring 


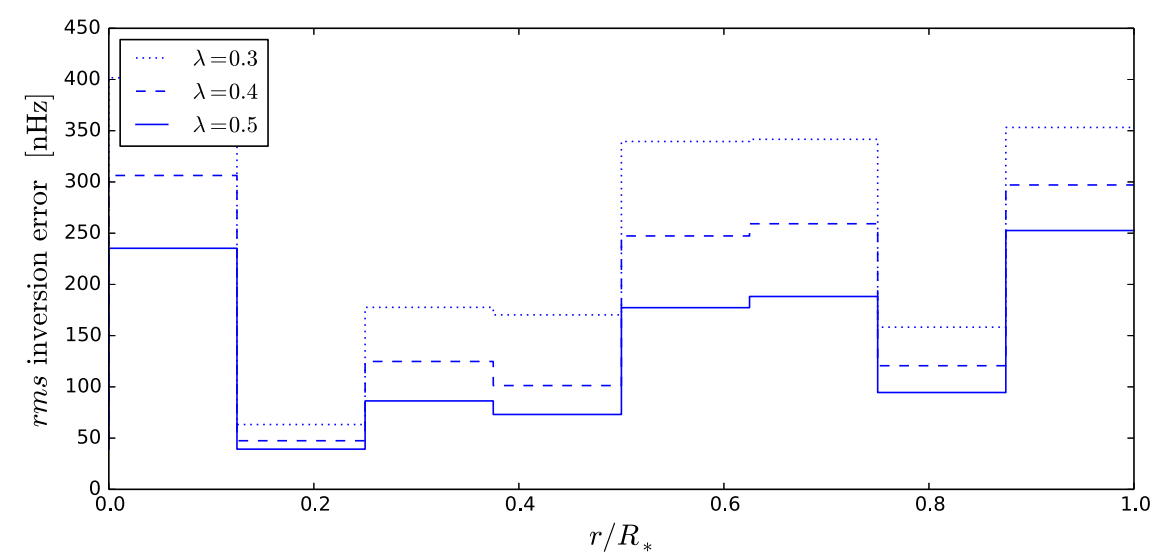

Figure 17. rms error incurred by RLS inversions with $\mu_{\mathrm{RLS}}=10^{-5}$ and $N=8$ computed from random profiles with various degrees of smoothing. See the text for details.

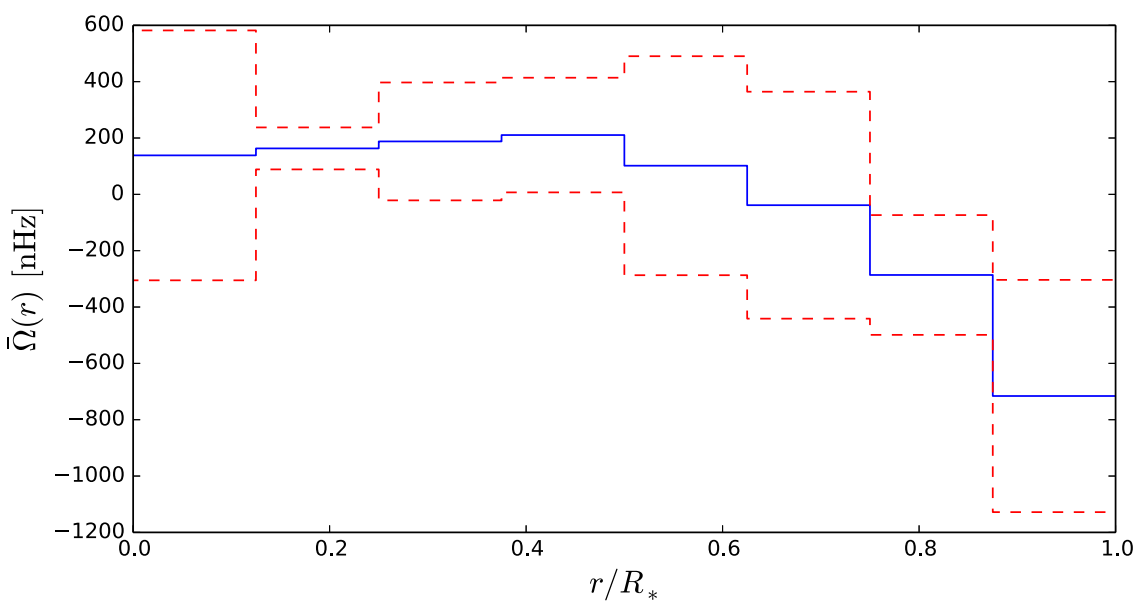

Figure 18. RLS inversion profile (solid line) of KIC $10526294\left(N=8, \mu_{\mathrm{RLS}}=10^{-5}\right)$. The error margins incorporate both the measurements errors (from Error Set 1 ) and the errors related to the inadequacy of the kernels to recover the "true" rotation profile. This latter error was estimated by using smooth random profiles with $\lambda=0.3$.

random profiles are strictly positive (or negative), they all involve at least one sign change along the radial coordinate.

This use of random profiles is also suitable to establish the "quality" of a set of kernels. To do this, we first take a random profile (the reference profile) and calculate its associated splittings via Equation (2). These splittings are then "inverted" and we compare the resulting profile with the reference profile. Some random noise could in principle be added to the splittings before attempting the inversion in order to simulate measurement errors, but this is unnecessary here since Equation (14) already properly describes the effect of the measurement variance on the inversion profiles. The differences between the inverted and reference profiles can therefore be attributed solely to the inadequacy of the kernel set to fully recover the solution.

To implement the above, we computed three sets of smooth random profiles $\left(\lambda=0.3,0.4,0.5\right.$, respectively, and with $10^{6}$ profiles each). We then rescaled the amplitude of each individual profile so as to make the corresponding splittings have a mean that equals the mean of the observed splittings. After discarding those profiles whose splittings had mixed signs, we proceeded to perform the inversions $\left(\mu_{\mathrm{RLS}}=10^{-5}\right)$. We computed inverted profiles with $N=8$ intervals of radial resolution and compared them with the reference profiles (integral-averaged over the same radial intervals). The standard errors at each radial interval calculated from all the profiles in the set are shown in Figure 17. We clearly see that the error becomes larger as the profiles have more variability. The radial locations where the errors are comparatively smaller coincide roughly with the locations where the $\mathbf{A}$ matrices have better localization (see Figure 26).

To conclude this section we present the inversion profile (from the real KIC 10526294 data) together with an overall $(1 \sigma)$ uncertainty (derived from both the measurement errors and the kernel error as explained above) in Figure 18. This profile represents a balance between good overall statistical measures and good localization properties, at least near the bottom of the radiative zone and close to the stellar surface. Note that a fully positive rotation profile is possible at the $2 \sigma$ level.

\section{SUMMARY AND CONCLUSION}

Numerical models and their pulsation properties (based on the MESA evolution code and the GYRE pulsation code) have allowed us to obtain kernels of oscillation modes whose frequencies closely match the identified zonal dipole mode frequencies of the B8V star KIC 1052629419 (Pápics 
et al. 2014; Moravveji et al. 2015). Based on these kernels, we computed the rotational profiles explaining the detected rotationally split dipole mode frequencies by assuming different functional forms (constant, linear, two-zone, and three-zone). We also performed RLS and SOLA inversions and implemented a Monte Carlo approach to obtain an approximate rotational profile and to estimate the errors incurred by the inversion process. We relied on the optimal equilibrium model found so far for this pulsator (Moravveji et al. 2015; Model 1), but other seismically derived equilibrium models were also examined and lead to qualitatively similar results.

While the most likely rotation profiles depend on assumptions made about the functional form of the profile, we were able to constrain the average rotation rate near the overshoot region to be about $163 \pm 89 \mathrm{nHz}$, a value supported by almost all of the rotational models we considered. Toward the surface of the star, our results are less constrained since they are sensitive to the a priori assumptions on the shape of the rotational profile. If a smooth and continuous profile is assumed, then our results point to a mild counter-rotating region in the envelope toward the surface of the star rotating at frequency $-717 \pm 412 \mathrm{nHz}$ with the sign change occurring around $r \sim 0.7 R_{*}$. On the other hand, if we assume a discontinuous two-zone profile, then we find an outer envelope rotating about six times slower than the overshoot region at $49 \mathrm{nHz}$. The averaging kernel associated with the outer zone of this two-zone model leads to a weighted average over most of the radiative envelope. The best counter-rotating profiles from inversion, when averaged over the radiative zone using this outer zone averaging kernel, lead to rotation rates entirely consistent between the two models.

We performed model comparisons based on the AIC as well as the leave-one-out cross-validation technique, which are both better suited than the reduced $\tilde{\chi}^{2}$ when comparing the performance of models that are not nested, as is the case for the models we considered in this study. Both methods give preference to inversion models with mild counter-rotation in the radiative envelope at the $1 \sigma$ level. The Monte Carlo simulations, fully independent of the above, are consistent with such a result. Current stellar structure models have so far not considered this type of physical ingredient.

Following the first rough estimates of $\Omega_{\text {core }} / \Omega_{\text {envelope }}$ for three core-hydrogen burning $\mathrm{B}$ stars prior to the asteroseismology space era (Aerts et al. 2003; Pamyatnykh et al. 2004; Briquet et al. 2007), the recent studies by Kurtz et al. (2014) and Saio et al. (2015) made the first high-precision asteroseismic measurement of surface-to-core rotation in two $\sim 1.5 M_{\odot}$ main-sequence hybrid heat-driven pulsators from four years of Kepler photometry. They found the star KIC 11145123 to have slightly faster envelope than core rotation and an average rotation period of $\sim 100$ day, while KIC 9244992 has slightly faster core than envelope rotation and an average rotation period of $\sim 65$ day. The authors deduced these results from the measured rotationally split g-mode triplets and p-mode triplets and quintuplets without relying on forward seismic modeling of the zonal modes as we have done in the present work. Our study of the $3.2 M_{\odot}$ main-sequence B-type star KIC 10526294 hints to an envelope whose inner rotation rate is opposite to its outer rate with a small factor ranging from $\sim-0.06$ to $\sim-0.2$ taking into account the uncertainties, while the star has a depth-averaged rotation period of about 186 day. In these three cases, even after taking into account that rotation rates at stellar birth have been largely overestimated (e.g., Zwintz et al. 2014), a strong and efficient mechanism must have been at work to slow down these stars' rotation after their birth. Moreover, an efficient mechanism must be active to transport angular momentum within the star. Internal gravity waves (IGWs) could be viable as such a mechanism. Indeed, numerical simulations based on IGWs for a $3 M_{\odot}$ star by Rogers et al. (2013) have shown that such waves can transfer angular momentum on short timescales and over the appropriate distances in stars with a convective core and a radiative envelope. Additionally, the study by Rogers et al. (2013) led to the conclusion that IGWs can lead to either a slightly faster envelope than core rotation, or an outer envelope rotating opposite to the inner regions. This mechanism thus could be the natural cause of the observational results on the rotational properties of KIC 10526294 , KIC 11145123, and KIC 9244992.

The type of rotation profile found for KIC 10526294 and KIC 11145123 is not achieved in any standard stellar evolutionary scenario. A similar but much stronger discrepancy between models and observations occurs for the core rotation of red giants (e.g., Cantiello et al. 2014). In our next step, we plan not only to perform similar studies for OB-type stars with various stellar parameters, but we will also investigate how the stellar structure, and in particular the density profile, behaves during the evolution of the star in the presence of the most likely rotation profiles we found in this study, testing new physical ingredients such as IGWs that have not yet been included to describe the physics in the radiative envelope of massive stars. Only an extension of the sample of stars with seismic inversion treated with appropriate statistical model selection and coupled to an iterative procedure to upgrade the input physics can deliver a meaningful improvement in the stellar models. Our study is the first step in this direction for massive stars.

The authors thank Profs. Geert Molenberghs and Marc Aerts of the Center for Statistics, University of Hasselt, Belgium, for enlightening discussions and valuable advice on statistical model selection. They also acknowledge detailed comments from a referee which helped them to present the results in a more consistent way. Furthermore, they are grateful to the Kepler team and everybody who has contributed to making this mission possible. Funding for the Kepler Mission was provided by NASA's Science Mission Directorate. The research leading to these results has received funding from the Fund for Scientific Research of Flanders (FWO, grant No. G.0728.11). E.M. is the beneficiary of a postdoctoral grant from the Belgian Federal Science Policy Office (Belspo) co-funded by the Marie Curie Actions FP7-PEOPLE-COFUND2008 No. 246540 MOBILITY GRANT from the European Commission. Funding for the Stellar Astrophysics Centre is provided by The Danish National Research Foundation (grant DNRF106). The research is supported by the ASTERISK project (ASTERoseismic Investigations with SONG and Kepler) funded by the European Research Council (grant agreement No. 267864). 

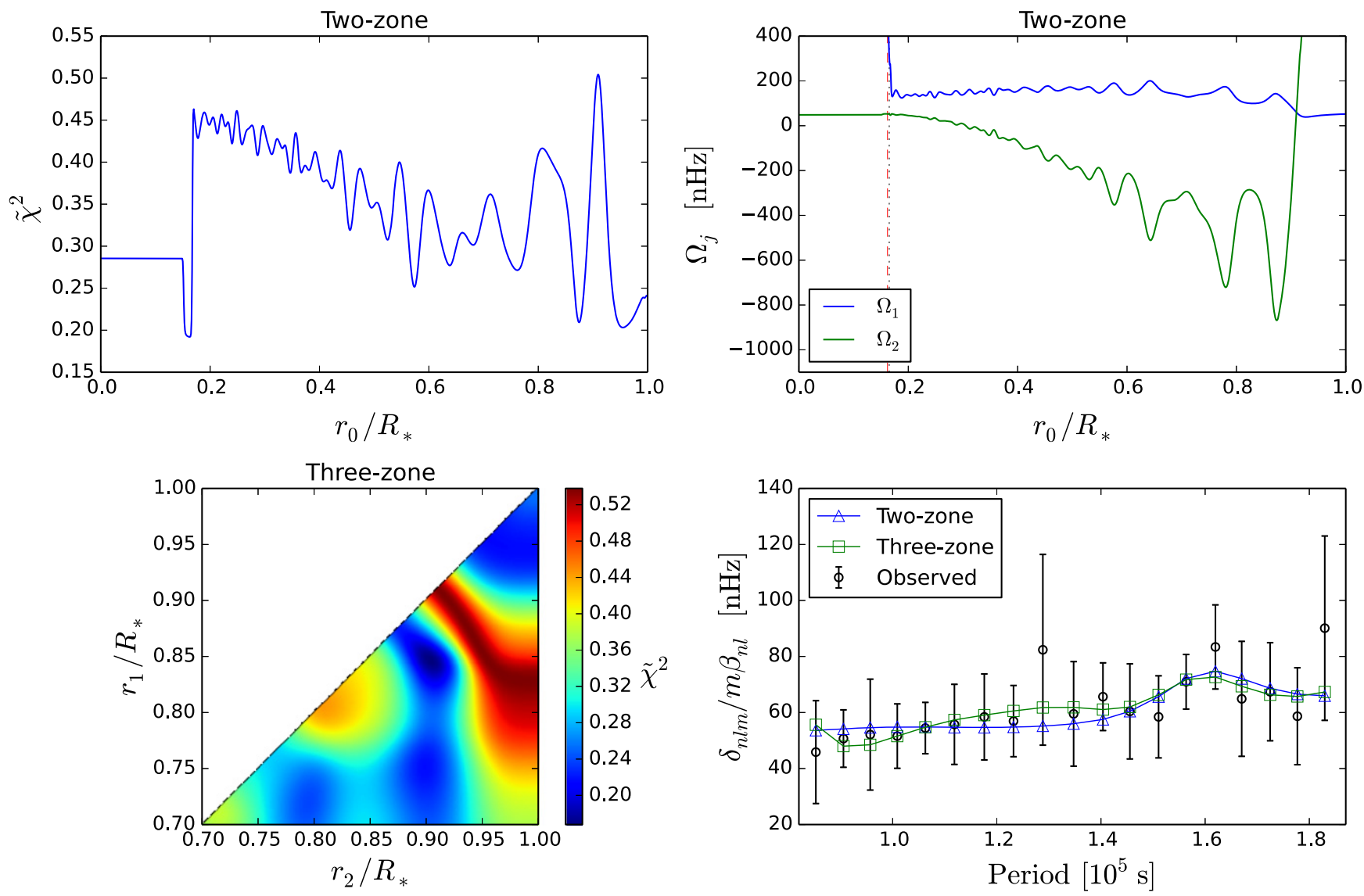

Figure 19. Same as Figure 8 with the exception that Error Set 2 has been used. The vertical dashed line in the top right panel marks the location of the minimum of $\tilde{\chi}^{2}$ for the two-zone model.

Table 4

Same as Table 3, but Using the Observation Uncertainties from Error Set 2

\begin{tabular}{lcccc}
\hline \hline Rotation Profile & rms Error $(\mathrm{nHz})$ & $\nu$ & $\tilde{\chi}^{2}$ & AICc \\
\hline Constant & 11.85 & 18.00 & 0.45 & 12.82 \\
Linear & 11.78 & 17.00 & 0.36 & 13.67 \\
Linear+ + & 11.66 & 16.00 & 0.45 & 18.05 \\
Two-zone & 9.61 & 16.00 & 0.19 & 13.93 \\
Three-zone & 8.44 & 15.00 & 0.17 & 17.13 \\
RLS, $N=8$ & 9.17 & 15.93 & 0.17 & 13.83 \\
RLS, $N=14$ & 10.29 & 16.09 & 0.26 & 14.66 \\
SOLA, $N=8$ & 9.46 & 14.30 & 0.22 & 20.80 \\
SOLA, $N=14$ & 7.59 & 11.48 & 0.15 & 35.90 \\
\hline
\end{tabular}

Table 5

Same as Table 3, but Excluding the Three Most Asymmetric Splittings, i.e., Those with Periods Near $0.96 \times 10^{5} \mathrm{~s}, 1.29 \times 10^{5} \mathrm{~s}$, and $1.83 \times 10^{5} \mathrm{~s}$

\begin{tabular}{lcrrr}
\hline \hline Rotation Profile & rms Error $(\mathrm{nHz})$ & $\nu$ & \multicolumn{1}{c}{$\tilde{\chi}^{2}$} & AICc \\
\hline Constant & 9.07 & 14.00 & 25.07 & 355.9 \\
Linear & 7.22 & 13.00 & 8.55 & 119.4 \\
Linear+ & 8.29 & 12.00 & 22.08 & 277.0 \\
Two-zone & 4.95 & 12.00 & 1.69 & 32.27 \\
Three-zone & 5.16 & 11.00 & 0.53 & 22.46 \\
RLS,$N=8$ & 4.84 & 11.29 & 0.63 & 22.33 \\
RLS,$N=14$ & 4.77 & 11.20 & 2.35 & 42.00 \\
SOLA, $N=8$ & 5.86 & 9.45 & 0.86 & 34.48 \\
SOLA, $N=14$ & 3.58 & 7.21 & 0.40 & 53.45 \\
\hline
\end{tabular}

Note. The single $m=+1$ mode with period near $1.78 \times 10^{5} \mathrm{~s}$ was also excluded. Error set 1 is used.
Table 6

Same as Table 5, but Using the Observation Uncertainties from Error Set 2

\begin{tabular}{lcccc}
\hline \hline Rotation Profile & rms Error $(\mathrm{nHz})$ & $\nu$ & $\tilde{\chi}^{2}$ & AICc \\
\hline Constant & 8.95 & 14.00 & 0.47 & 11.63 \\
Linear & 7.05 & 13.00 & 0.32 & 12.36 \\
Linear+ + & 8.27 & 12.00 & 0.47 & 17.66 \\
Two-zone & 4.91 & 12.00 & 0.14 & 13.67 \\
Three-zone & 4.70 & 11.00 & 0.13 & 18.11 \\
RLS,$N=8$ & 4.96 & 12.15 & 0.14 & 13.11 \\
RLS,$N=14$ & 5.85 & 12.39 & 0.24 & 13.42 \\
SOLA, $N=8$ & 6.04 & 10.99 & 0.23 & 19.27 \\
SOLA, $N=14$ & 3.49 & 8.59 & 0.10 & 34.55 \\
\hline
\end{tabular}

APPENDIX A

\section{RESULTS FOR ERROR SET 2 AND/OR FOR LIMITED TRIPLET SETS}

Figures 19-25 and Tables 4-6 show the results obtained when Error Set 2 is used as well as the results when considering only a limited set of splittings.

\section{APPENDIX B THE A MATRICES}

As mentioned in Section 6, the matrix A gives an indication of how well the inversion profile recovers the true profile (in the ideal case of no measurement error in the 

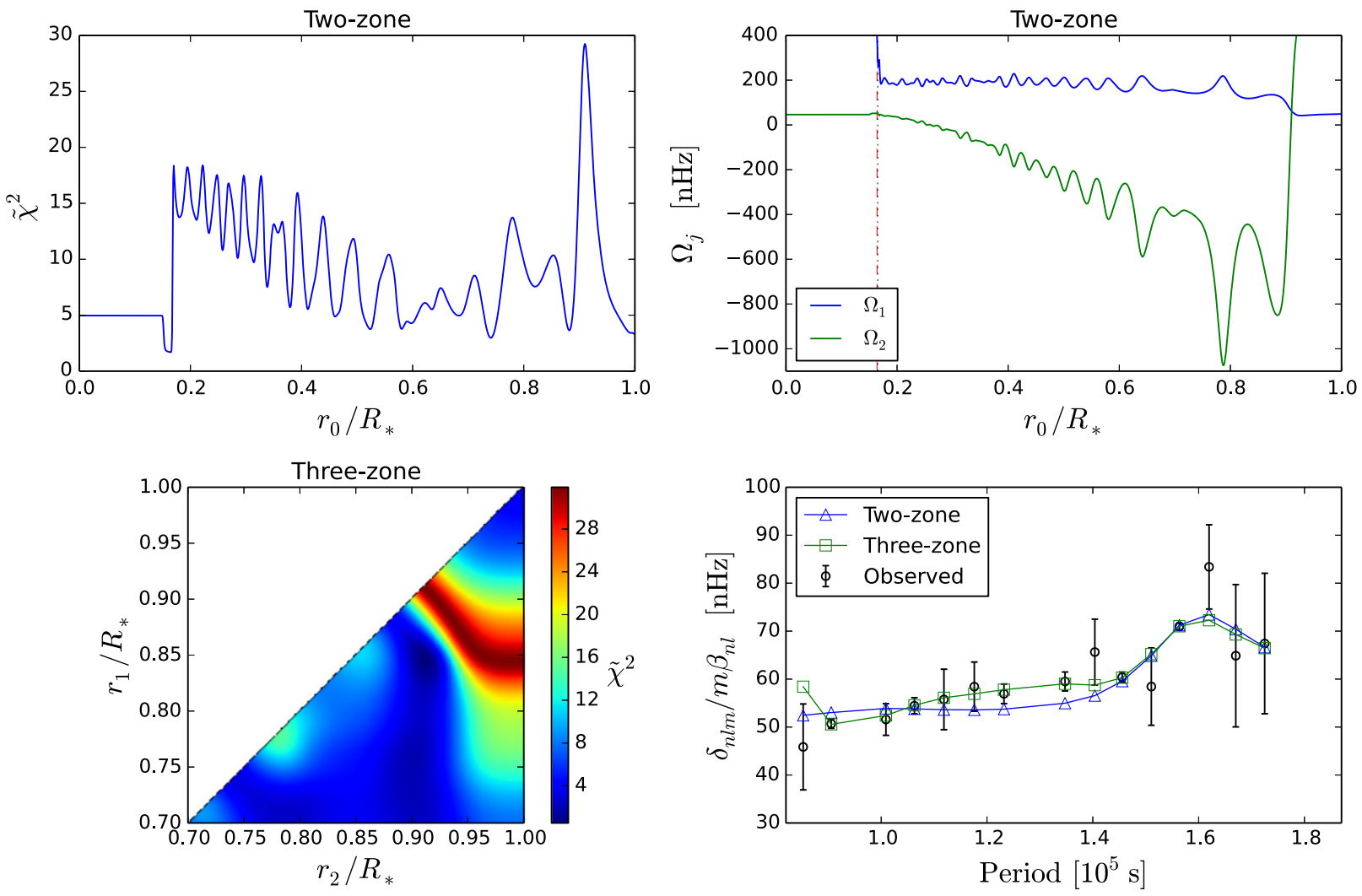

Figure 20. Same as Figure 19 with the exception that the three most asymmetric splittings have been excluded from the $\tilde{\chi}^{2}$ minimization, i.e., those with periods near $0.96 \times 10^{5} \mathrm{~s}, 1.29 \times 10^{5} \mathrm{~s}$, and $1.83 \times 10^{5} \mathrm{~s}$. The single $m=+1$ splitting with period near $1.78 \times 10^{5} \mathrm{~s}$ was also excluded. Error Set 1 has been used.
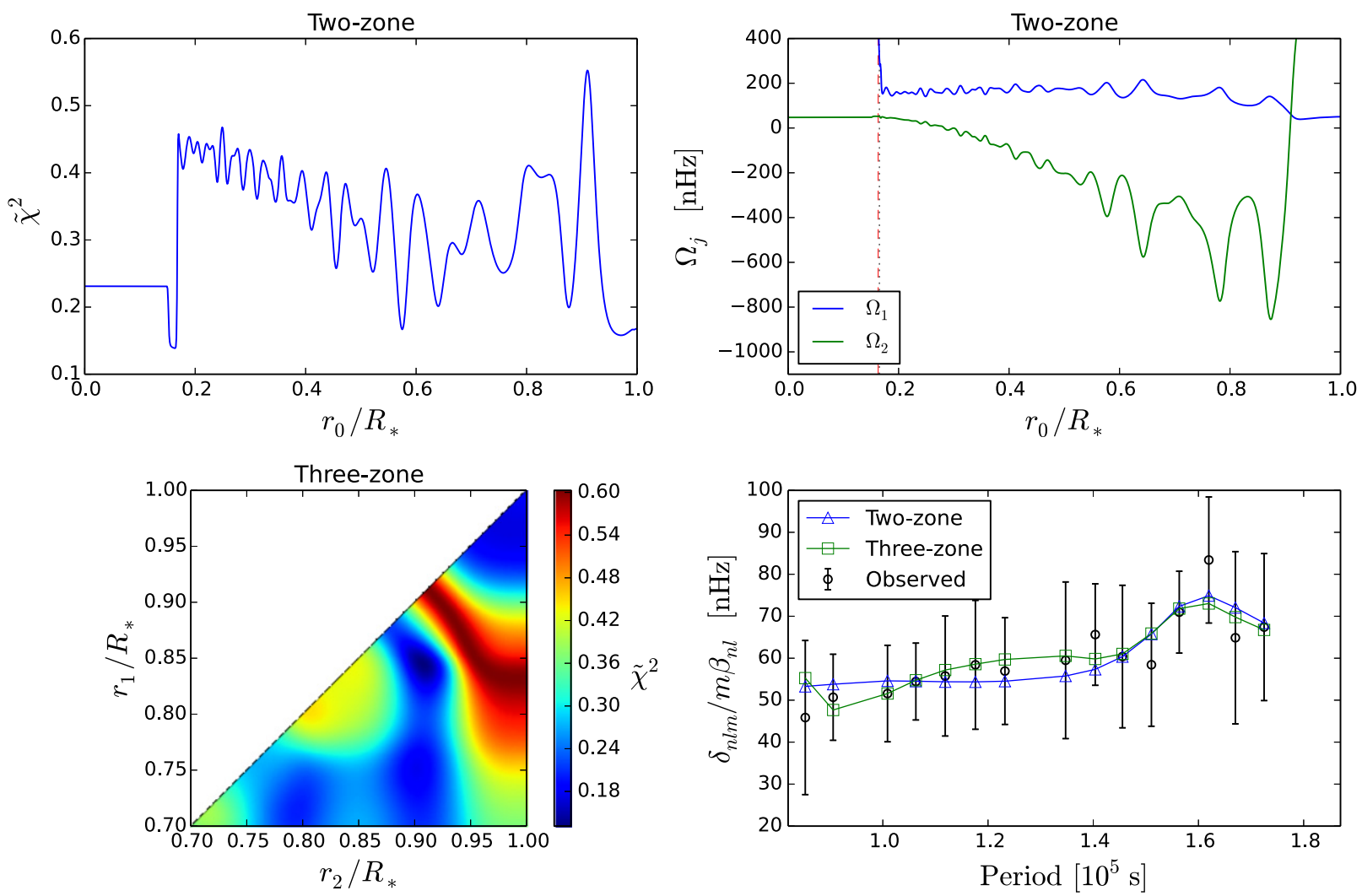

Figure 21. Same as Figure 20, i.e., omitting the three most asymmetric modes and the single $m=+1$ splitting, but this time using Error Set 2 . 

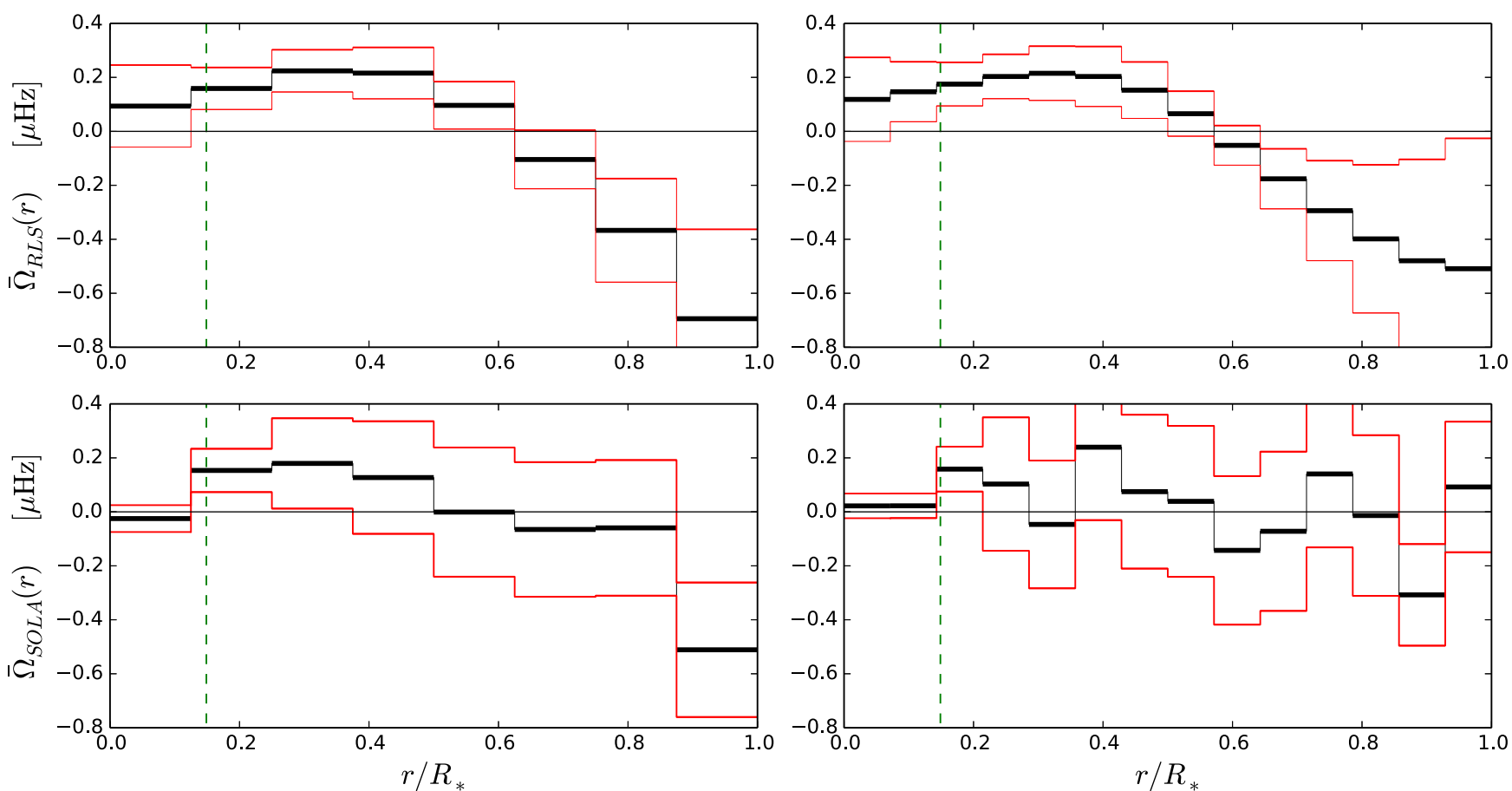

Figure 22. Same as Figure 10 with the exception that Error Set 2 has been used.
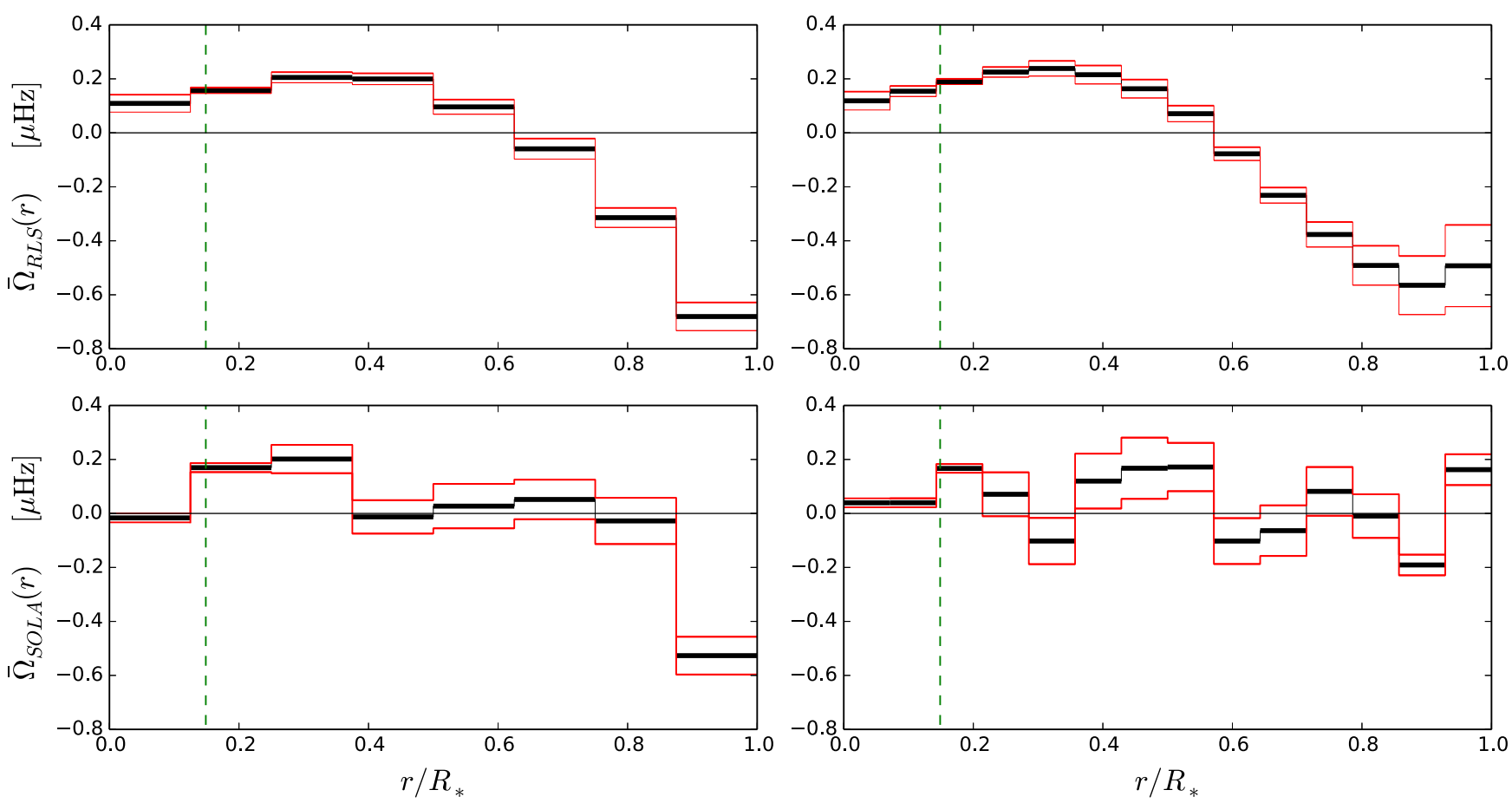

Figure 23. Same as Figure 10 but this time excluding the three most asymmetric splittings, i.e., those with periods near $0.96 \times 10^{5} \mathrm{~s}, 1.29 \times 10^{5} \mathrm{~s}$, and $1.83 \times 10^{5} \mathrm{~s}$. The single $m=+1$ mode with period near $1.78 \times 10^{5} \mathrm{~s}$ was also excluded from the computation.

splittings). Using the best model from Moravveji et al. (2015), we can see this fact at work very clearly in both Figures 26 and 27 as the respective $\mu$ parameter is varied. The more A resembles the identity matrix, the better the reconstruction is, thus providing a qualitative assessment of the inversion.

\section{APPENDIX C}

\section{AVERAGING KERNELS IN THE CONTINUOUS LIMIT}

The RLS and SOLA inversions behave very differently when the resolution is increased. In this section's experiments, we kept $\mu_{\mathrm{RLS}}=10^{-5}$ and $\mu_{\mathrm{SOLA}}=10^{-2}$ fixed but used three different resolutions, $N=14,28,5000$. From the 

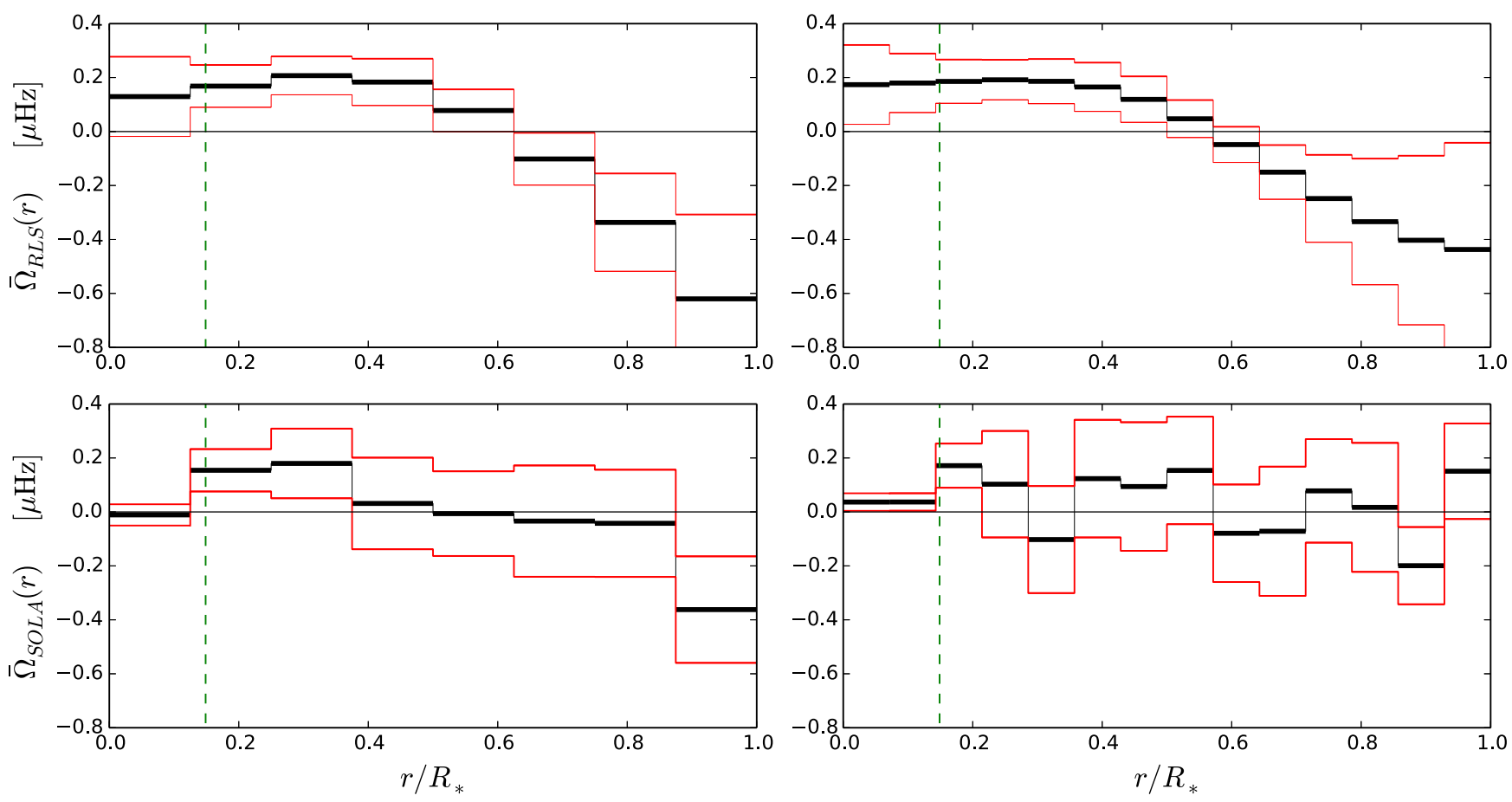

Figure 24. Same as Figure 23 with the exception that Error Set 2 has been used.
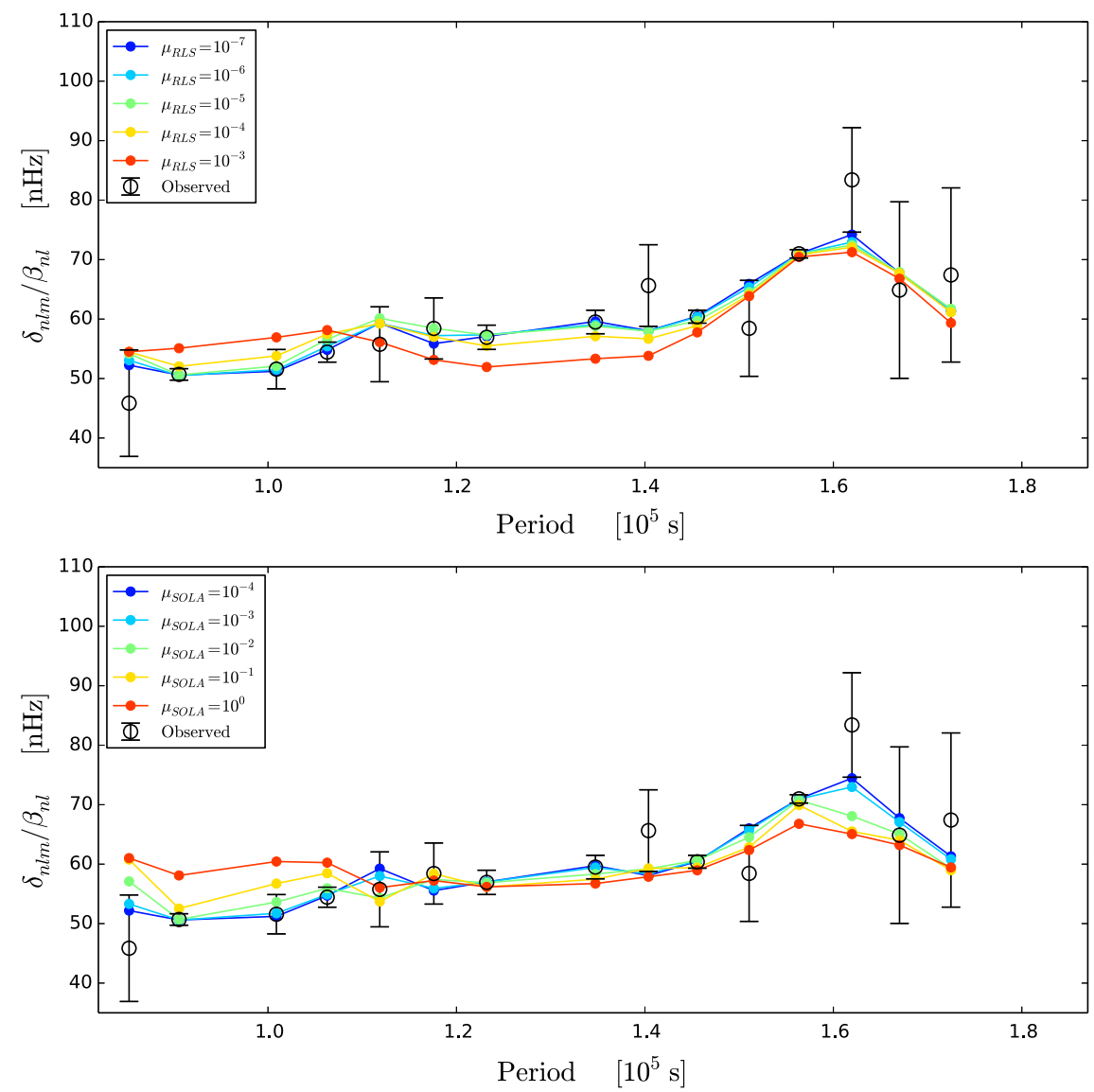

Figure 25. Same as Figure 11 but excluding the three most asymmetric splittings, i.e., those with periods near $0.96 \times 10^{5} \mathrm{~s}, 1.29 \times 10^{5} \mathrm{~s}$, and $1.83 \times 10^{5} \mathrm{~s}$. The single $m=+1$ mode with period near $1.78 \times 10^{5} \mathrm{~s}$ was also excluded from the computation. 
$\mu_{R L S}=10^{-7}$
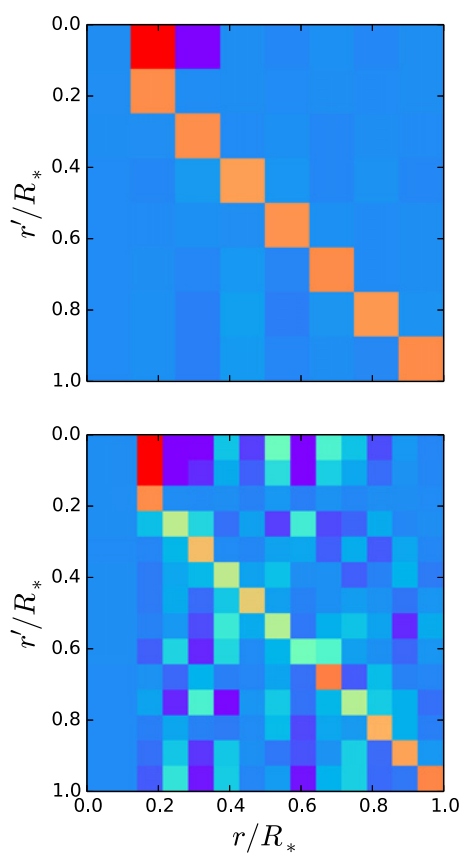

$\mu_{R L S}=10^{-6}$
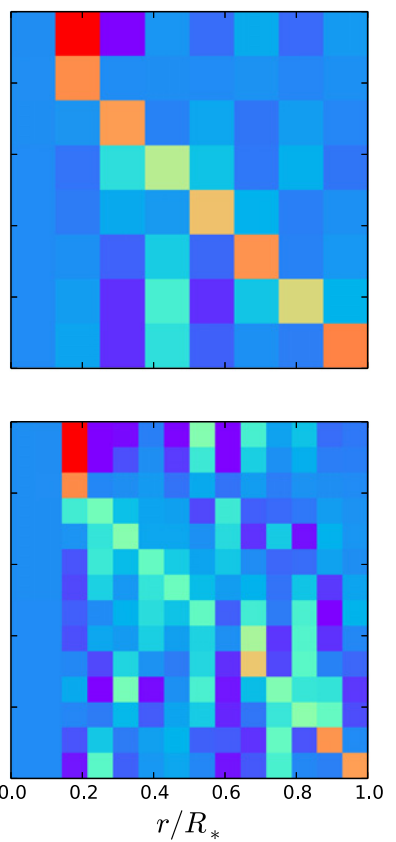

$\mu_{R L S}=10^{-5}$
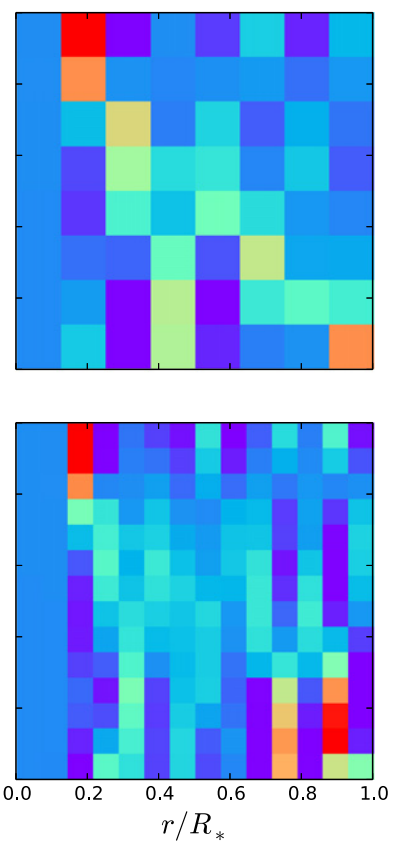

$\mu_{R L S}=10^{-4}$
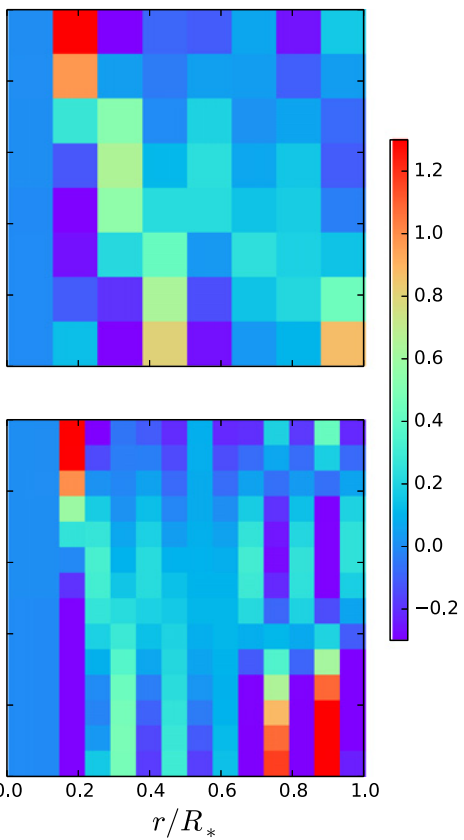

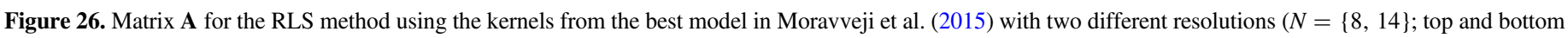

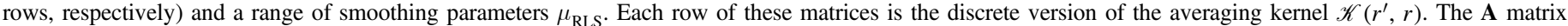

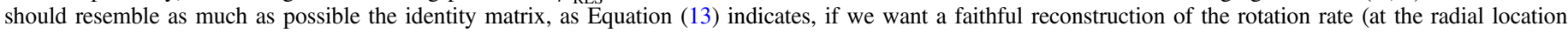

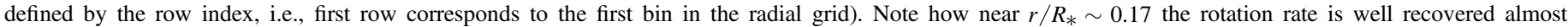
independently of the choice of $N$ or $\mu_{\text {RLS }}$.
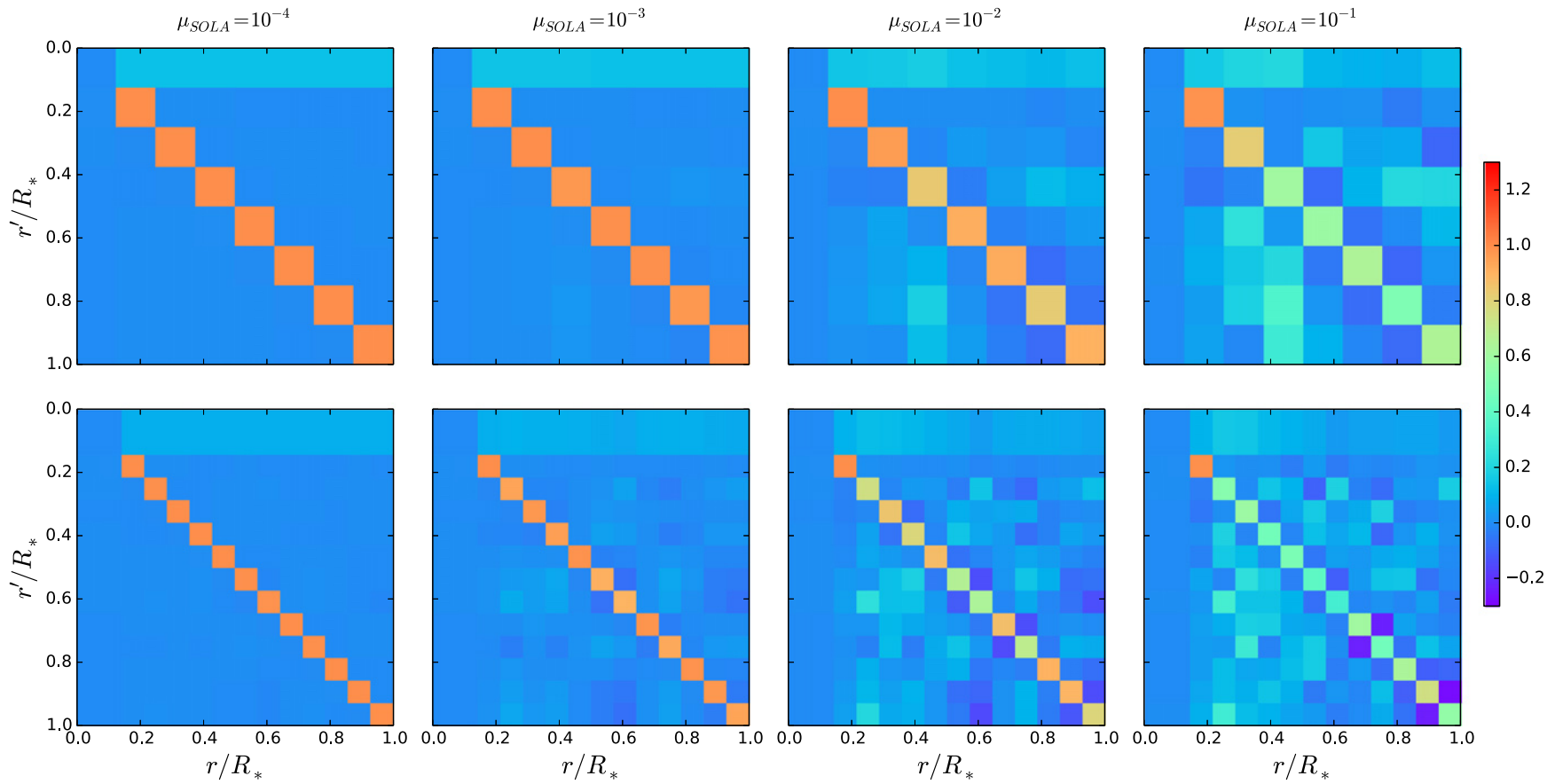

Figure 27. Same as in Figure 26, but using the SOLA method. The matrices are very well localized except at higher resolutions and higher $\mu_{\text {SOLA }}$. At $\mu_{\text {SOLA }}=10^{-2}$ and $N=8$, there is a small amount of leakage from regions close to $r=0.4 R_{*}$ into the outer radial bins $\left(r^{\prime} \sim 0.9 R_{*}\right)$. We estimate from this that the rotation rate there is overestimated by the inversion by about $3 \%$.

viewpoint of the inversion, it is possible to increase the resolution beyond the number of observations $M$ since regularization keeps the effective number of fitted parameters below $M$. Here, we do not include the variances on the inversions in the discussion. We have chosen Error Set 1 as the uncertainties on the splittings.

In Figure 28, we show the resulting inversion profiles. There are two radial locations where most of the rotation rates 


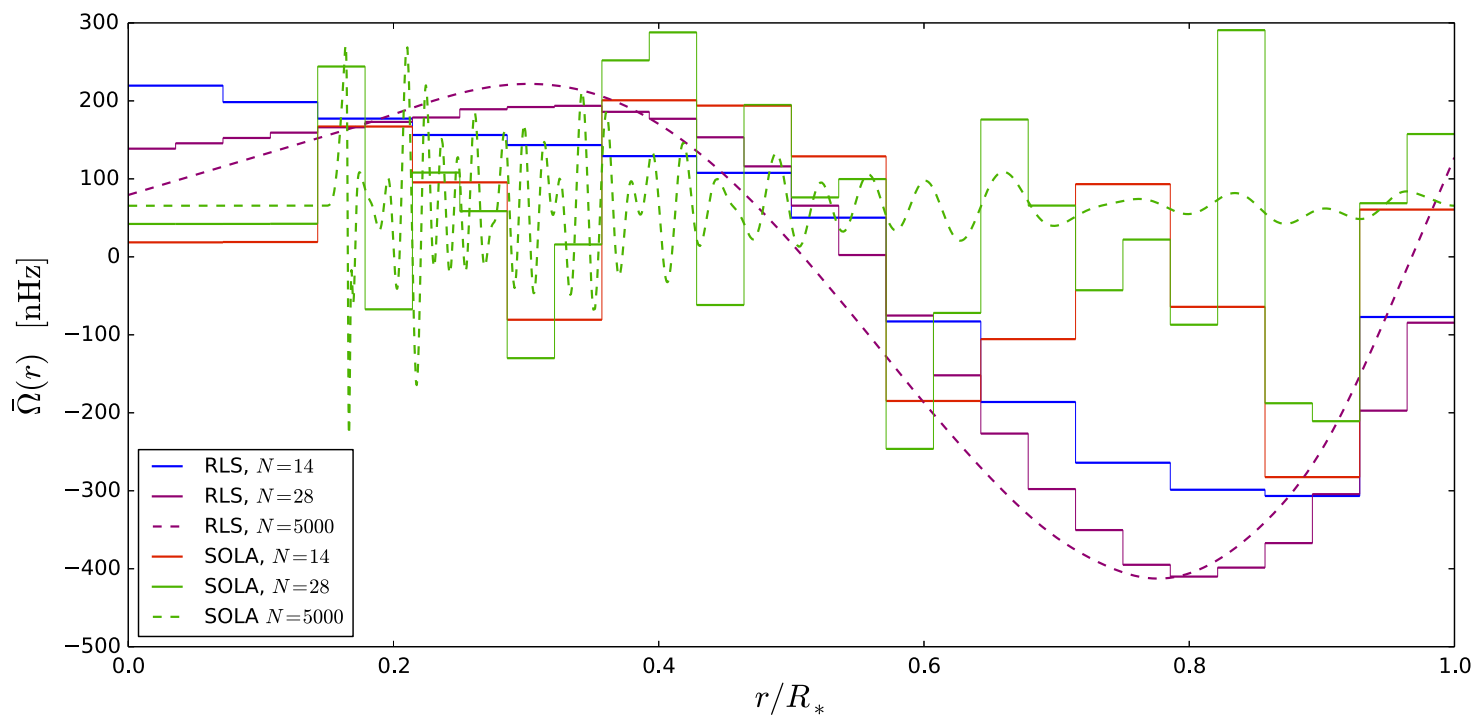

Figure 28. Rotation profiles from the RLS $\left(\mu_{\mathrm{RLS}}=10^{-5}\right)$ and SOLA $\left(\mu_{\mathrm{SOLA}}=10^{-2}\right)$ methods and three different resolutions $N=14,28,5000$. The inversions are based on the kernels from the best model of Moravveji et al. (2015). The measurement uncertainties are taken from Error Set 2.
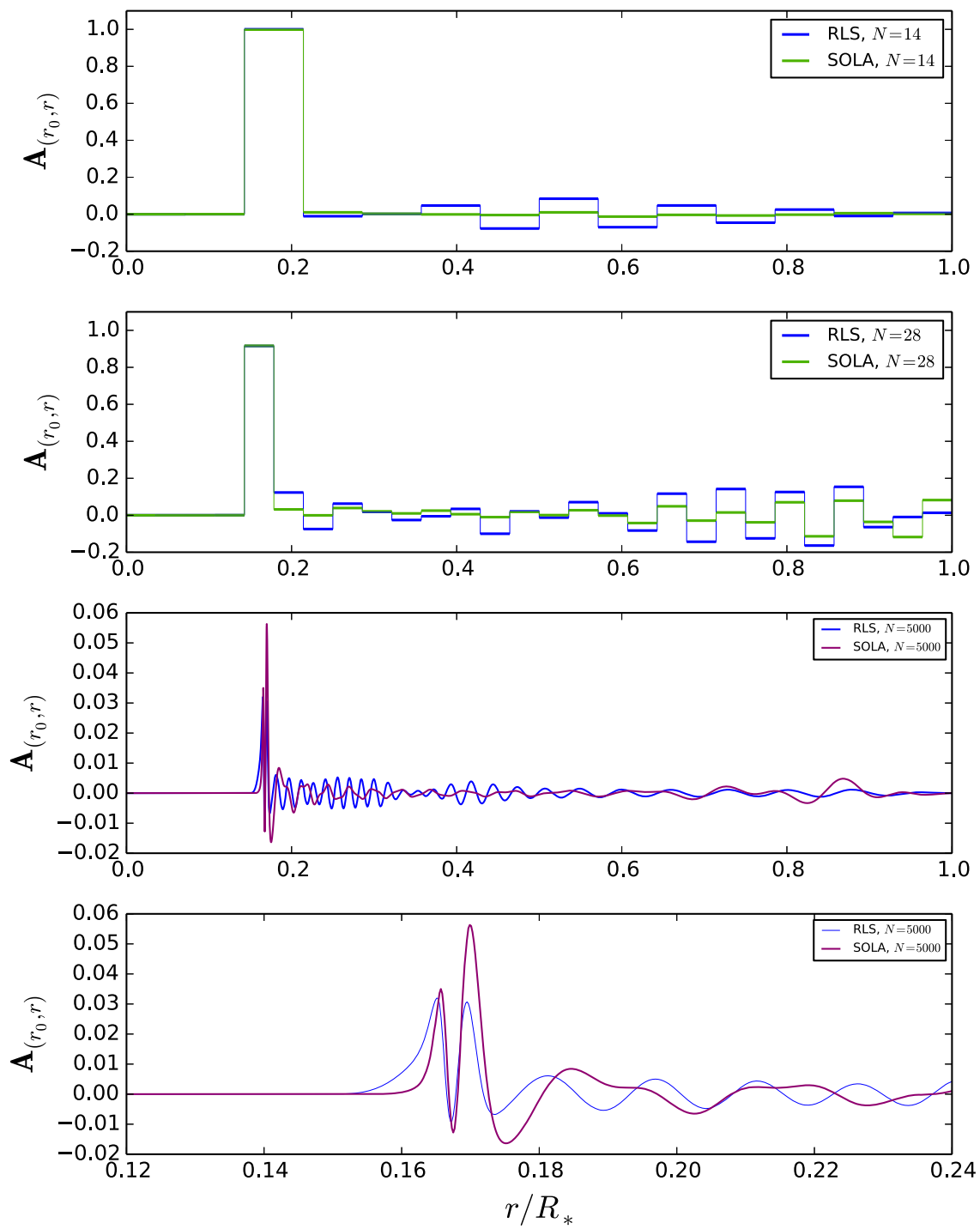

Figure 29. Row of $\mathbf{A}$ corresponding to $r_{0}=0.17$ at three resolutions (top three plots). The bottom plot is a zoom of the one immediately above. 

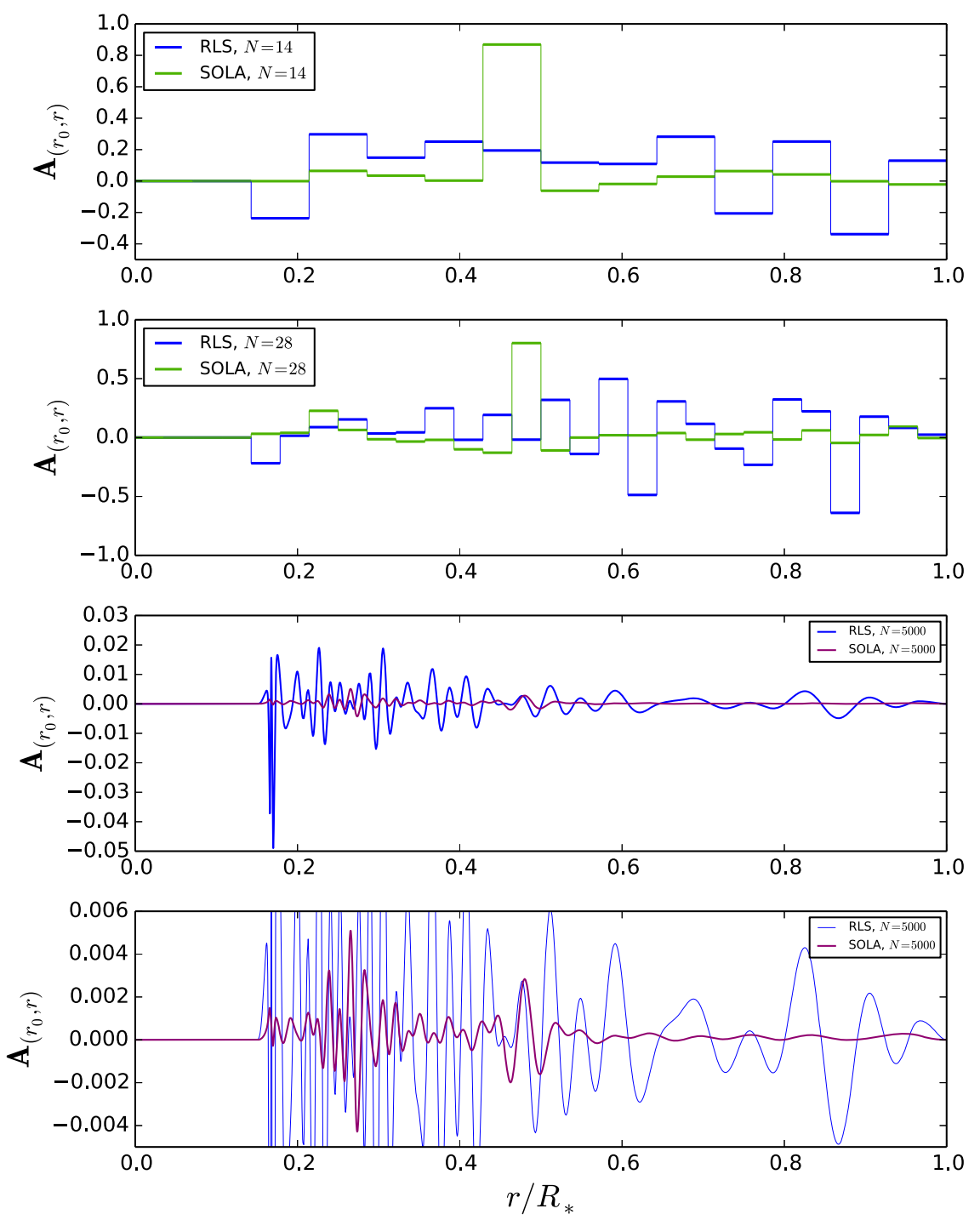

Figure 30. Same as Figure 29, but for $r_{0}=0.48$.

roughly coincide. One is at $r \sim 0.17$ and the other is at $r \sim 0.92$ where the rotation values are not too far from each other, except for the SOLA inversions with $N=28,5000$.

Let us examine the averaging kernels from three selected radial locations $r_{0}=0.17 R_{*}, r_{0}=0.48 R_{*}$, and $r=0.92 R_{*}$ as shown in Figures 29-31, respectively. From the figures, we see that for $r_{0}=0.17 R_{*}$, all of the averaging kernels are indeed generally well behaved, and so we expect inferences for this location to be consistent. At $r_{0}=0.48$ (Figure 30), the localization is acceptable as long as $N$ is low. Closer to the stellar surface, at $r_{0}=0.92$ (Figure 31 ), the situation is similar although the RLS kernels degrade considerably when $N=28$.

From these figures, we conclude that consistent inferences can be found using either the RLS or SOLA methods if the resolution is kept low, i.e., $N \lesssim 14$. Such a resolution also implies a sensible model comparison through the AICc (see Table 3), while larger $N$ and small smoothing parameters would lead to an effective number of degrees of freedom $\nu<1$ and strongly negative AICc values, implying overfitting from the viewpoint of the model comparison.

\section{APPENDIX D \\ TESTING RLS INVERSIONS WITH A SYNTHETIC PROFILE}

The following test is to check that the counter-rotation profiles are not produced by some undesired property of the RLS inversion methods. We take the optimum two-zone model from Section 5 and smooth it using the "low pass" filter with correlation length $\lambda=0.3$ described in Section 7. This is taken as the "actual" rotational profile, which does not exhibit counter-rotation. Subsequently, we calculate the associated exact rotational splittings via Equation (2). To each of these 19 splittings we add random noise sampled from a Gaussian distribution with zero mean and the same standard deviation as the actual measurement errors (Error Set 1). We set $N=8, \mu_{\mathrm{RLS}}=10^{-5}$ as used for the RLS inversions in the main text and proceed to calculate the inversion profile.

At each radial bin, we compare the inversion value with the integral average of the "actual" profile over the same radial bin. This gives us a direct estimate of the inversion error. By repeating this process a large number of times, we can obtain well-defined statistics (we used $10^{7}$ iterations). Figure 32 shows 
The Astrophysical Journal, 810:16 (26pp), 2015 September 1
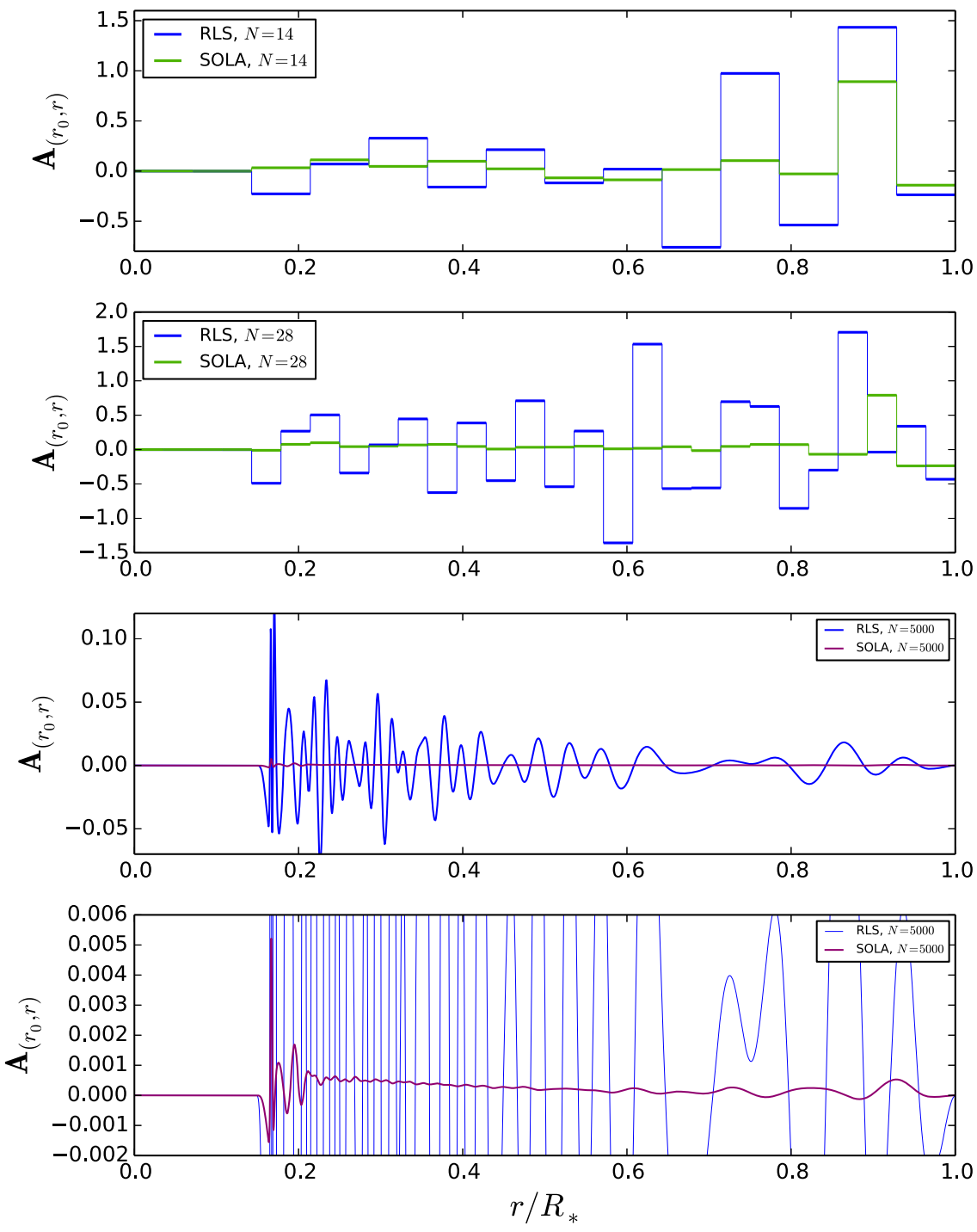

Figure 31. Same as Figure 30, but for $r_{0}=0.92$.

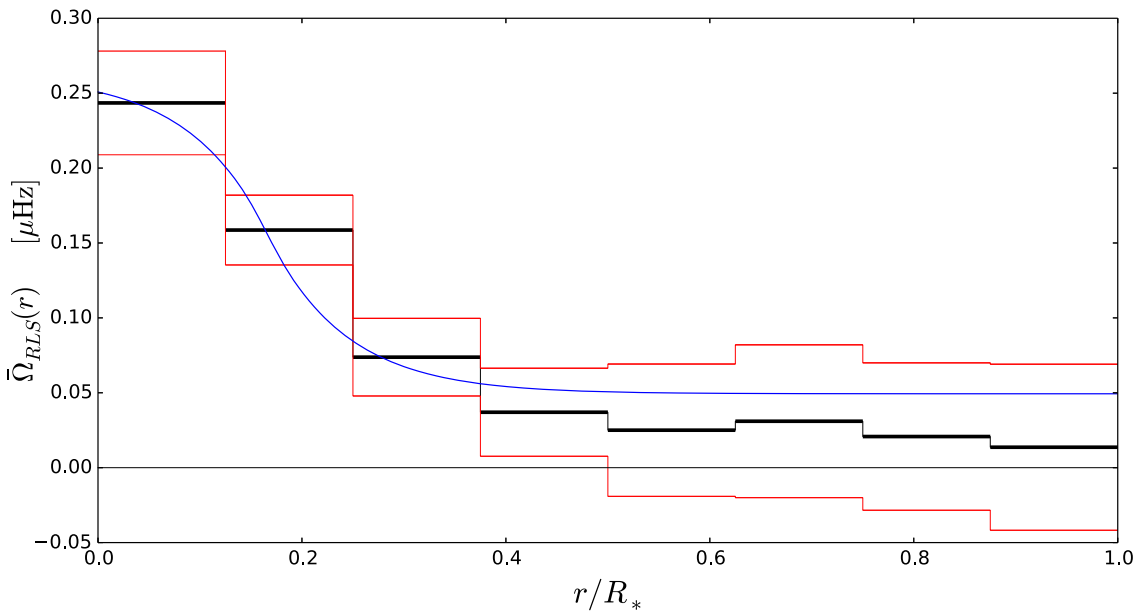

Figure 32. Smoothed-out two-zone model (blue, correlation length $\lambda=0.3$ ) and the recovered profile from RLS inversion (black, $\mu_{\mathrm{RLS}}=10^{-5}$ ). Error bounds are in red. 
the "actual" profile in blue, the recovered profile in black, and the estimated $1 \sigma$ uncertainty range in red. The RLS method does a good job of recovering the actual profile, which always occurs within the errors. Some inversion profiles must counterrotate mildly since the error region extends below zero in the outer half of the star. However, given the errors, we do not find a counter-rotating profile in this case.

\section{REFERENCES}

Aerts, C., Christensen-Dalsgaard, J., \& Kurtz, D. W. 2010, Asteroseismology, Astronomy and Astrophysics Library (Berlin: Springer)

Aerts, C., Thoul, A., Daszyńska, J., et al. 2003, Sci, 300, 1926

Beck, P. G., Hambleton, K., Vos, J., et al. 2014, A\&A, 564, A36

Beck, P. G., Montalban, J., Kallinger, T., et al. 2012, Natur, 481, 55

Boyd, S., \& Vandenberghe, L. 2004, Convex Optimization (Cambridge: Cambridge Univ. Press)

Briquet, M., Morel, T., Thoul, A., et al. 2007, MNRAS, 381, 1482

Burnham, K., \& Anderson, D. 2002, Model Selection and Multimodel Inference: A Practical Information-Theoretic Approach (Berlin: Springer)

Cantiello, M., Mankovich, C., Bildsten, L., Christensen-Dalsgaard, J., \& Paxton, B. 2014, ApJ, 788, 93

Charpinet, S., Fontaine, G., \& Brassard, P. 2009, Natur, 461, 501

Christensen-Dalsgaard, J. 2002, RvMP, 74, 1073

Córsico, A. H., Althaus, L. G., Kawaler, S. D., Miller Bertolami, M. M., \& García-Berro, E. 2012, ASP Conf. Ser. 462, Progress in Solar/Stellar Physics with Helio- and Asteroseismology, ed. H. Shibahashi, M. Takata, \& A. E. Lynas-Gray (San Francisco, CA: ASP), 176

Craig, I., \& Brown, J. 1986, Inverse Problems in Astronomy A Guide to Inversion Strategies for Remotely Sensed Data (Bristol: Hilger)

Degroote, P., Aerts, C., Baglin, A., et al. 2010, Natur, 464, 259

Degroote, P., Briquet, M., Catala, C., et al. 2009, A\&A, 506, 111

Deheuvels, S., Doan, G., Goupil, M. J., et al. 2014, A\&A, 564, A27
Deheuvels, S., García, R. A., Chaplin, W. J., et al. 2012, ApJ, 756, 19

Eggenberger, P., Montalbán, J., \& Miglio, A. 2012, A\&A, 544, L4

Gough, D. 1985, SoPh, 100, 65

Hasan, S. S., Zahn, J.-P., \& Christensen-Dalsgaard, J. 2005, A\&A, 444, L29

Hastie, T., Tibshirani, R., \& Friedman, J. 2009, The Elements of Statistical Learning: Data Mining, Inference, and Prediction (Berlin: Springer)

Hurvich, C. M., \& Tsai, C.-L. 1989, Biometrika, 76, 297

Kawaler, S. D., \& Bradley, P. A. 1994, ApJ, 427, 415

Kawaler, S. D., Sekii, T., \& Gough, D. 1999, ApJ, 516, 349

Kuhn, H. W., \& Tucker, A. W. 1951, Proc. Second Berkeley Symp. on Mathematical Statistics and Probability (Berkeley, CA: Univ. California Press), 481

Kurtz, D. W., Saio, H., Takata, M., et al. 2014, MNRAS, 444, 102

Ledoux, P. 1951, ApJ, 114, 373

Maeder, A. 2009, Physics, Formation and Evolution of Rotating Stars (Berlin: Springer)

Moravveji, E., Aerts, C., Papics, P. I., Andres Triana, S., \& Vandoren, B. 2015, A\&A, 580, A27

Mosser, B., Goupil, M. J., Belkacem, K., et al. 2012, A\&A, 548, A10

Pamyatnykh, A. A., Handler, G., \& Dziembowski, W. A. 2004, MNRAS, 350, 1022

Pápics, P. I., Moravveji, E., Aerts, C., et al. 2014, A\&A, 570, A8

Paxton, B., Bildsten, L., Dotter, A., et al. 2011, ApJS, 192, 3

Paxton, B., Cantiello, M., Arras, P., et al. 2013, ApJS, 208, 4

Pijpers, F. P., \& Thompson, M. J. 1994, A\&A, 281, 231

Rogers, T. M., Lin, D. N. C., McElwaine, J. N., \& Lau, H. H. B. 2013, ApJ, 772,21

Saio, H., Kurtz, D. W., Takata, M., et al. 2015, MNRAS, 447, 3264

Thompson, M. J., Christensen-Dalsgaard, J., Miesch, M. S., \& Toomre, J. 2003, ARA\&A, 41, 599

Townsend, R. H. D., \& Teitler, S. A. 2013, MNRAS, 435, 3406

Triana, S. A., Zimmerman, D. S., Nataf, H.-C., et al. 2014, NJPh, 16, 113005

van Saders, J. L., \& Pinsonneault, M. H. 2013, ApJ, 776, 67

Zwintz, K., Fossati, L., Ryabchikova, T., et al. 2014, Sci, 345, 550 